Supporting Information for

\title{
Pulsed interleaved MINFLUX
}

Luciano A. Masullo ${ }^{1,2 \dagger}$, Florian Steiner ${ }^{3 \dagger}$, Jonas Zähringer ${ }^{3}$, Lucía F. Lopez ${ }^{1}$, Johann Bohlen ${ }^{3}$ Lars Richter $^{1,3}$, Fiona Cole ${ }^{3}$, Philip Tinnefeld ${ }^{3 *}$, Fernando D. Stefani $^{1,2 *}$

1 Centro de Investigaciones en Bionanociencias (CIBION), Consejo Nacional de Investigaciones Científicas y Técnicas (CONICET), Godoy Cruz 2390, C1425FQD, Ciudad Autónoma de Buenos Aires, Argentina

2 Departamento de Física, Facultad de Ciencias Exactas y Naturales, Universidad de Buenos Aires, Güiraldes 2620, C1428EHA, Ciudad Autónoma de Buenos Aires, Argentina

3 Department of Chemistry and Center for NanoScience, Ludwig-Maximilians-Universität München, Butenandtstr. 5-13 Haus E, 81377 München, Germany 


\section{Supplementary Information}

\section{Table of Contents}

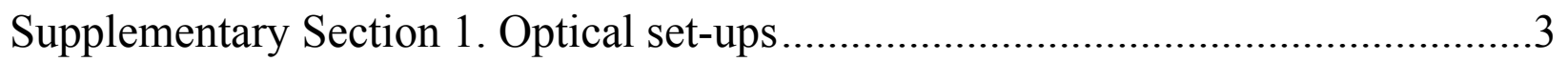

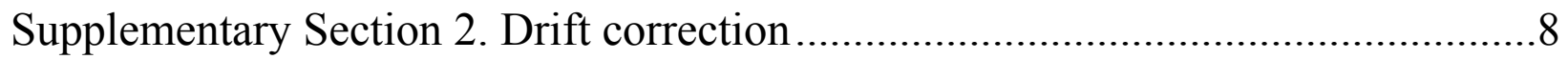

Supplementary Section 3. DNA-origami structure folding ....................................9

Supplementary Section 4. Sample preparation..................................................10

Supplementary Section 5. Determination of the excitation beam pattern and the

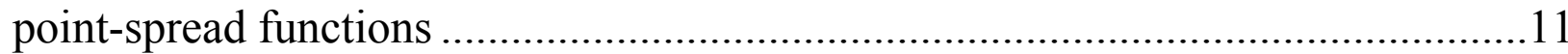

Supplementary Section 6. p-MINFLUX measurement ......................................12

Supplementary Section 7. Position estimator and Cramér-Rao bound ....................14

Supplementary Section 8. p-MINFLUX simulations ...........................................17

Supplementary Section 9. Effect of misalignment of the excitation beam pattern 18

Supplementary Section 10. Photon arrival times and crosstalk between detection

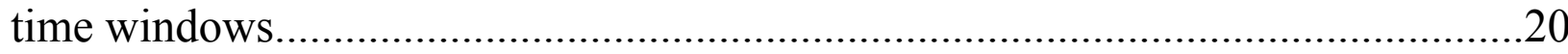

Supplementary Section 11. Localization time traces simulation............................23

Supplementary Table S1. Original DNA-origami staples sequences for experiments

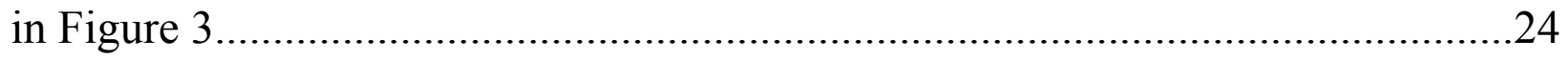

Supplementary Table S2. Functionalized DNA-origami staples sequences for

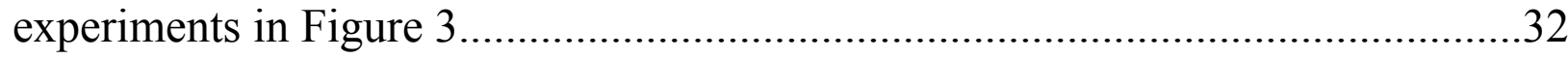

Supplementary Table S3. Original DNA-origami staples sequences for experiments in Figure 4.

Supplementary Table S4. Functionalized DNA-origami staples sequences for

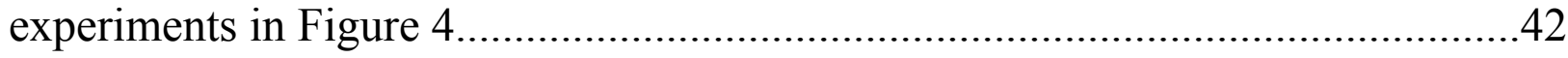

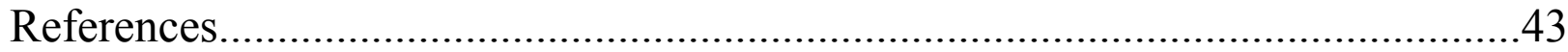




\section{Supplementary Section 1. Optical set-ups}

Two versions of the p-MINFLUX microscope were built and are fully functional. One in Prof. Fernando Stefani's laboratory at CIBION in Buenos Aires, Argentina, and the other in Prof. Philip Tinnefeld's laboratory at LMU in Munich, Germany.

A

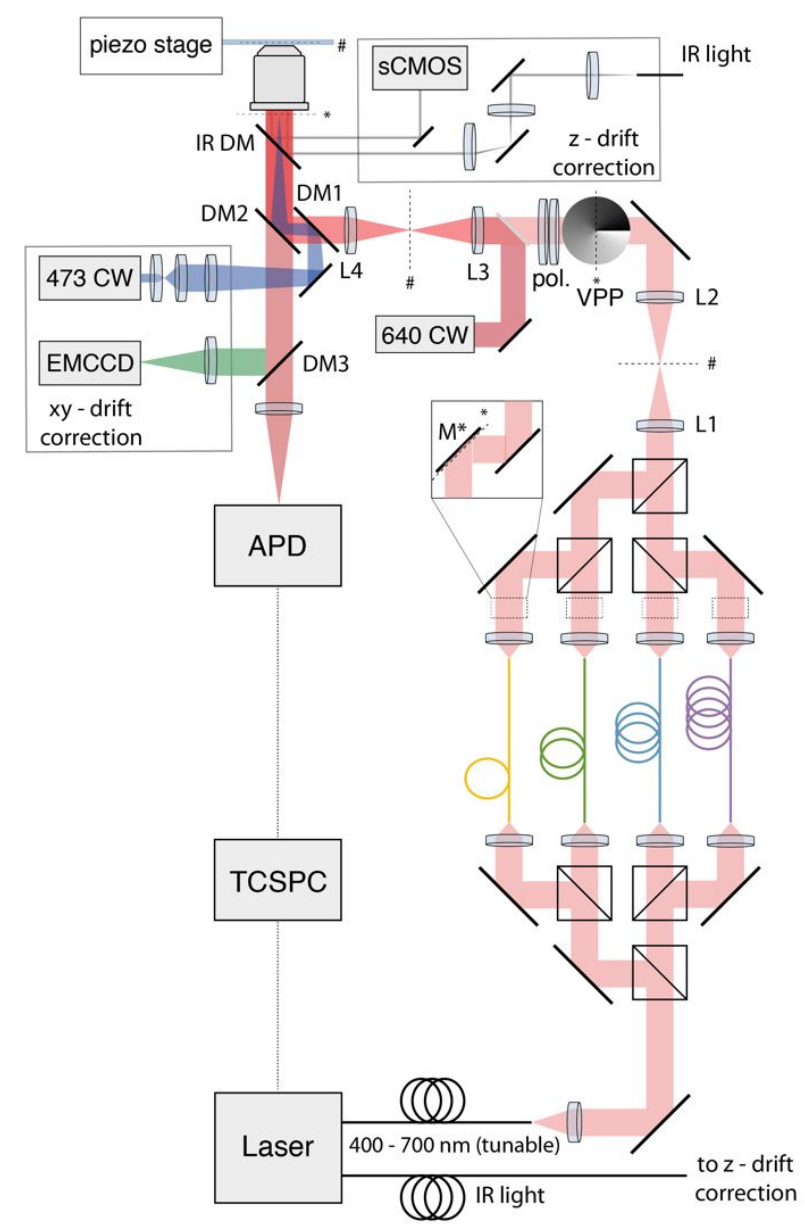

B

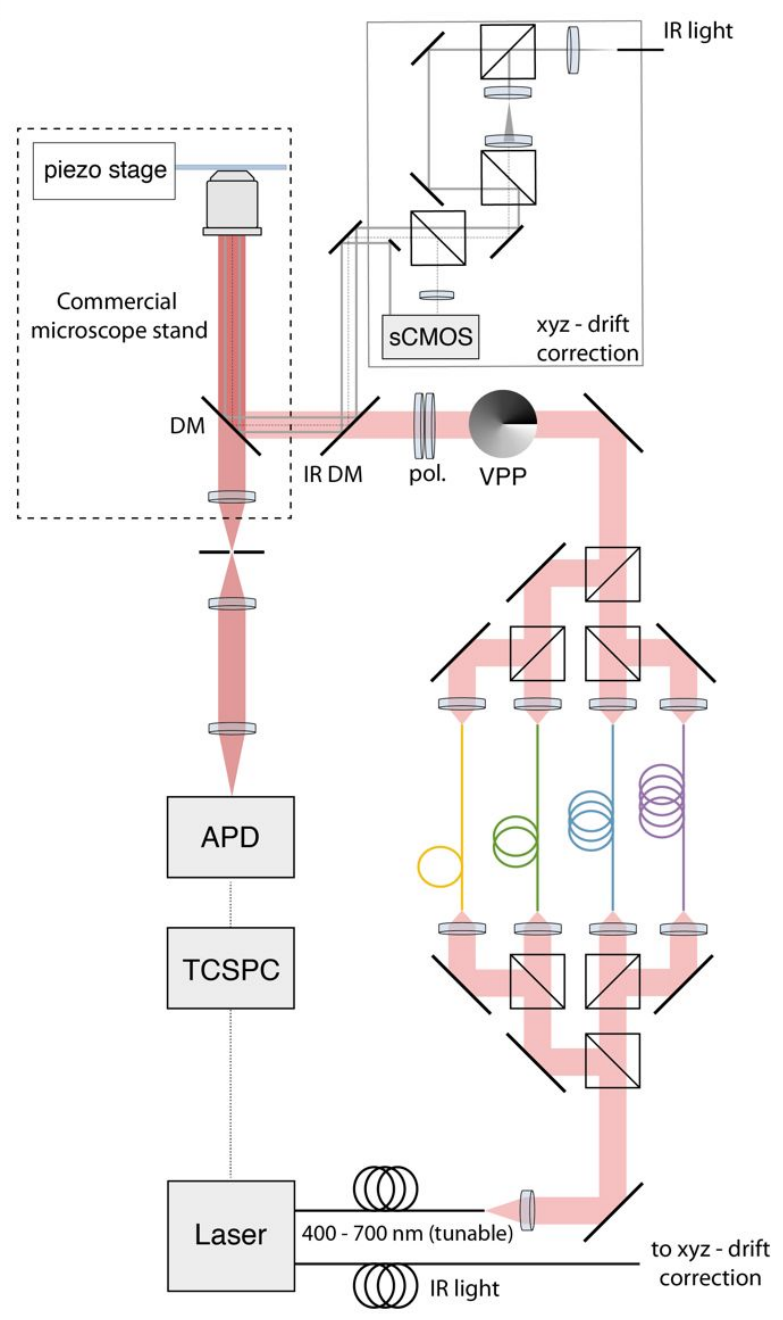

Supplementary Figure S1. A) p-MINFLUX setup built in Buenos Aires. Laser: Fianium SC 400-4 (NKT Photonics), 640 CW: Lambda mini Evo, RGB Photonics GmbH, 473 CW: GEM 473, Laser Quantum, piezo stage: NanoMax 300 with controller BPC303, Thorlabs Inc.), Lens 1 (L1), L2, L3, L4: AC254-300-A-ML (Thorlabs Inc.), VPP: V-633-10, Vortex Photonics, Polarizer (pol.): WPH05M-633 and WPQ05M-633, Thorlabs Inc.), Dichroic Mirror (DM1): ZT532/640RPC-UF, Chroma Technology Corporation, DM2: ZT532/640RPC, Chroma Technology Corporation, IR DM: T750SPXRXT-UF1, Chroma Technology Corporation, DM3: 
ZT532RDC, Chroma Technology Corporation, EMCCD: iXon DV887 (Andor), sCMOS: ThorCam DCC1240C (Thorlabs Inc.), Avalanche Photodiode (APD): SPCM-AQR-13 (PerkinElmer Optoelectronics), time-correlated single-photon-counting (TCSPC): PicoHarp 300 (PicoQuant $\mathrm{GmbH}$ ). B) p-MINFLUX setup built in Munich. Laser: SuperK Fianium FIU-15 (NKT Photonics GmbH), piezo stage: P733.3CD (Physik Instrumente (PI) GmbH), VPP: V-53220-1 (Vortex Photonics) pol.: WPQ05M-532 and WPH532M-532, Thorlabs GmbH, IR DM: ZT 785 SPXXR (Chroma Technology Corp.), DM: ZT532RDC - STED (Chroma Technology Corp.), sCMOS: Zelux, (Thorlabs GmbH), APD: SPCM-AQRH-16-TR (Excelitas Technologies GmbH \& Co. KG), TCSPC: HydraHarp 400, PicoQuant GmbH).

\section{Buenos Aires setup:}

Excitation. A supercontinuum laser (Fianium SC 400-4, NKT Photonics) is used for excitation with pulses of $\sim 80 \mathrm{ps}$ width (FWHM) and a repetition rate of $1 / \mathrm{T}=40 \mathrm{MHz}$. The emission spectrum ranges from $\lambda \sim 420 \mathrm{~nm}$ to $\lambda \sim 2000 \mathrm{~nm}$ and the output of the laser system is a collimated, unpolarized, beam. Infrared light with $\lambda>1064 \mathrm{~nm}$ is filtered out by a dichroic mirror (BLP01-1064R-25, Semrock Inc.). A small fraction of the IR light is sent to a custom-built, fast photodiode sensing the arrival of the laser pulses. The rest of the far-infrared light is dumped. Near-infrared light is split from the main beam (T750SPXRXT-UF1, Chroma Technology Corporation) and coupled into a multimode fiber to be used in the z-drift correction optics. From the remaining visible light, a $\sim 20 \mathrm{~nm}$ band around $642 \mathrm{~nm}$ is selected for fluorescence excitation with an interference optical filter (ZET642/20x, Chroma Technology Corporation).

The light intensity is controlled using a flipper with a mounted ND filter (MFF101/M and NDK01, Thorlabs Inc.) and a continuous, ND filter NDC-50C-2. The excitation beam is then split by a polarizing beam splitter (PBS) (CCM1-PBS251/M, Thorlabs Inc.). The two beams have orthogonal polarization states. Each of the beams is sent to a non-polarizing 50:50 beam splitter (CCM1-BS013/M, Thorlabs Inc.). This beam splitting system generates two pairs of beams with each pair sharing the orthogonal linear polarization.

The four beams are coupled into polarization-maintaining fibers (PM630-HP, Thorlabs Inc.). Each of the fibers has a different optical path length, approx. $1.3 \mathrm{~m}, 2.6 \mathrm{~m}, 3.9 \mathrm{~m}$, and $5.2 \mathrm{~m}$ which generate the $\mathrm{T} / 4$ time delay between pulses. The light coupled out of the fibers carries linear polarization. It is reflected with dielectric mirrors (BB1-E02, Thorlabs Inc.) into the beam combining system. The four beams are combined by three non-polarizing 50:50 beam splitters (CCM1-BS013/M, Thorlabs Inc.) used as beam combiners. The axes of linear polarization are matched by turning the fiber out-couplers (60FC, Schäfter + Kirchhoff GmbH).

The recombined beams are focused by a lens L1, $f=300 \mathrm{~mm}$ (AC254-300-A-ML, Thorlabs Inc.) to a plane conjugated to the back focal plane of the microscope objective. Lens L1 is the first out of four identical lenses forming a double 4f-system. Between L2 and L3 a VPP (V-633-10, Vortex Photonics) is placed in the conjugated plane to the back focal plane (BFP) of the objective. Conjugated planes are indicated with * and \# in Supplementary Figure S1. The VPP creates the doughnut-shaped focus in the focal plane of the oil-immersion objective (CFI Plan Fluor 100x, 
$\mathrm{NA}=1.4$, Nikon Instruments Inc.). Circular polarization is needed to create the minima of intensity in the center of the doughnut-shaped foci and is achieved by the combination of $\lambda / 2$ and $\lambda / 4$ waveplates (WPH05M-633 and WPQ05M-633, Thorlabs Inc.) which are positioned close to L3.

The four temporally-separated pulses share the doughnut excitation pattern. To align the four doughnut centers to the desired EBP positions, the mirrors $\mathrm{M}^{*}$ are employed. They are placed in the conjugate plane to VPP and BFP and allow to spatially shift the doughnut-shaped focus by changing their tilt angles without changing their lateral position relative to the VPP. This allows ensuring almost the same phase modulation in the Fourier plane for the four beams. Additionally, a pinhole can be placed into the conjugated plane of the sample before the VPP to clean-up the beams for possible distortions at the beam splitters used for recombination.

An auxiliary confocal system consisting of a $\mathrm{CW}$ diode laser (Lambda mini Evo, RGB Photonics $\mathrm{GmbH}$ ) emitting at $\lambda=640 \mathrm{~nm}$ was added to the setup to provide an excitation with a gaussian beam profile. Using a pellicle 92:8 beam splitter (BP108, Thorlabs Inc.), the collimated laser light is coupled into the excitation optical path.

Detection. The light emitted by the sample is collected by the objective. It passes a dichroic mirror (ZT532/640RPC, Chroma Technology Corporation) which reflects the excitation beams. Notch filters block the excitation light from the red excitation beams (ZET642NF, Chroma Technology Corporation), from the blue widefield excitation (ZET473NF, Chroma Technology Corporation), and the reflected infrared beam (NF808-34, AHF Analysentechnik AG).

The fluorescence emission is focused by a tube lens (AC254-50-A-ML, Thorlabs Inc.) onto an avalanche photodiode (APD) (SPCM-AQR-13, PerkinElmer Optoelectronics). The APD detects photons with $90 \%$ quantum efficiency at $\lambda=650 \mathrm{~nm}$. It provides a single photon timing resolution of 350 ps (FWHM) and typically exhibits 150 dark counts per second. The digital signal from the fluorescence photons is sent from the APD to a TCSPC unit (PicoHarp 300, PicoQuant). The internal timer of the TCSPC unit correlates the photon detection signal from the APD to the signal from the photodiode which senses the arrival of the excitation laser pulse. For every counted photon, it returns an absolute time from the beginning of the measurement and the time elapsed since the last laser pulse. The temporal resolution of the TCSPC unit is $8 p s$ and the dead time after a photon detection is reported by the manufacturer to be $<95 \mathrm{~ns}$.

Drift correction. The optical system providing z-stabilization employs the near-infrared light coupled into a multimode fiber. After attenuation and clean-up (ET705/72M and T750SPXRXTUF1, Chroma Technology Corporation), the IR light is guided to the objective. The collimated beam enters the objective at the edge of the back focal aperture. After reflection from the samplecoverslip interface, the IR light leaves the objective parallel to the incident IR beam. The reflected light passes a tube lens (AC254-150-A-ML, Thorlabs Inc.) and is focused on a CMOS camera (ThorCam DCC1240C, Thorlabs Inc.). Through a calibration with the piezo stage, we determined that the $\mathrm{z}$ position of the slide can be tracked with a precision better than $10 \mathrm{~nm}$ and actively corrected with a feedback loop.

The lateral stabilization of the sample is realized tracking bright green fluorescent beads (FluoSpheres, 0,2 $\mu \mathrm{m}$, Nile Red, Thermo Fisher Scientific). As displayed in Supplementary Figure 
S1, a widefield configuration is employed for illuminating the beads at (GEM 473, Laser Quantum). The fluorescence emission is reflected by a dichroic mirror (ZT647RDC, Chroma Technology Corporation) and filtered (ZET473NF, Chroma Technology Corporation). The light is focused by a tube lens (AC254-300-A-ML, Thorlabs Inc.) and imaged by an EMCCD camera (iXon DV887, Andor). The processing of the data for the xy-stabilization is explained in Supplementary Section S2.

Setup control. The core of the instrument control system is a data acquisition board (DAQ board) (ADwin Gold II, Jäger $\mathrm{GmbH}$ ) which serves as an analog-digital and digital-analog converter. The ADwin allows programming its output channels for the direct control of the connected devices from a computer. The ADwin receives and processes the raw photon count data from the APD which are used for creating an image from the confocal scan. Additionally, it connects to the piezo controller and the electromechanical shutters (MediaLas STP 8xl, spectrabeam.de) closing/opening the different beam paths. The piezo stage (NanoMax 300 with controller BPC303, Thorlabs Inc.) translates the sample in all three dimensions with a resolution of $5 \mathrm{~nm}$ when running in closed-loop mode.

The setup control software constitutes an open-source instrumentation project called PyFLUX, freely available at https://github.com/lumasullo/pyflux and https://github.com/cibionconicet/pyflux. Further details on specific Python instrumentation and updated versions of the code are available at this repository.

\section{Munich setup:}

Excitation. A supercontinuum laser (SuperK Fianium FIU-15, NKT Photonics GmbH, Germany) is used at $19.5 \mathrm{MHz}$ repetition rate as a light source in combination with a tunable bandpass filter (SuperK VARIA, NKT Photonics GmbH, Germany) to select the desired wavelength range (527 $-537 \mathrm{~nm})$ in the visible light spectrum followed by an additional clean-up filter (520/35 Brightline HC, Semrock Inc., USA). The unpolarized light from the laser source is split by a polarizing beam splitter cube (PBS251, Thorlabs GmbH, Germany) into two beams of orthogonal polarizations. Each of the beams is further split by a non-polarizing 50:50 beam splitter cube (BS013, Thorlabs $\mathrm{GmbH}$, Germany). This beam splitting system generates two pairs of beams with each pair sharing the orthogonal linear polarization. The resulting four laser beams are coupled into polarizationmaintaining single-mode fibers (PM-S405-XP, Thorlabs $\mathrm{GmbH}$, Germany) of lengths $2.0 \mathrm{~m}, 4.6 \mathrm{~m}, 7.1 \mathrm{~m}$ and $9.7 \mathrm{~m}$ such that the time delay between the beams after the fiber is $\sim 12.5$ ns $(=\mathrm{T} / 4)$. The four beams are collimated after the fibers with an achromatic lens (AC254-035-A, Thorlabs GmbH, Germany) and recombined by using three 50:50 beam splitter cubes (BS013, Thorlabs GmbH, Germany). The overlay of the beams can be adjusted to obtain the required arrangement of laser foci in the object plane. The axes of linear polarization are matched by turning the fiber out-couplers (Thorlabs GmbH, Germany). Subsequently, the linearly polarized laser beams pass a combination of a quarter- and a half-wave plate (WPQ05M-532 and WPH532M532, Thorlabs $\mathrm{GmbH}$, Germany) to make them circularly polarized. A vortex phase plate (V-53220-1, Vortex Photonics, Germany) is then used to introduce the phase modulation necessary to 
generate the donut-shaped foci. The beams are guided into the back entrance of the microscope body (IX83, Olympus Deutschland GmbH, Germany), reflected on a dichroic mirror (ZT532RDC - STED, Chroma Technology Corp., USA) and focused with an objective (UPLSAPO100XO/1.4, Olympus Deutschland GmbH, Germany) onto the sample plane.

Detection. The fluorescence light is collected with the same objective and transmitted through the dichroic mirror, focused via an Olympus tube lens onto a pinhole $(120 \mu \mathrm{m}$, Owis, Germany), collimated with an achromatic lens (AC254-150-A, Thorlabs GmbH, Germany) and focused with a second achromatic lens (AC127-025-A, Thorlabs GmbH, Germany) to the chip of an avalanche photodiode (SPCM-AQRH-16-TR, Excelitas Technologies GmbH \& Co. KG, Germany) after filtering the remaining scattered light from the laser with suitable interference optical filters (785 SP EdgeBasic, Semrock Inc., USA, 2x 582/75 Brightline HC, Semrock Inc. USA). The digital signal from the APD is sent to a TCSPC unit (HydraHarp 400, PicoQuant GmbH, Germany).

Drift correction. To measure and correct for sample drift during the measurements, the IR output of the variable bandpass filter is used. A beam of wavelength between 850 and $900 \mathrm{~nm}$ is selected with optical filters (875/50 bandpass, Edmund Optics $\mathrm{GmbH}$ ), coupled into a single-mode fiber (780HP, Thorlabs GmbH, Germany), outcoupled and collimated. This beam is then split with a 50:50 beam splitter cube (BS014, Thorlabs $\mathrm{GmbH}$, Germany) and combined again after inserting a lens system (ACN254-040-B, AC254-150-B, Thorlabs GmbH, Germany) into one of the two paths that focuses the beam to the back focal plane of the objective (dotted line) to create a widefield illumination at the sample plane. This beam is used for xy drift correction where the position of fiducial markers is localized during the measurement. The collimated IR beam is focused onto the sample plane at an oblique angle to achieve a $\mathrm{z}$ position-dependent spot at the detector and use this for $\mathrm{z}$ drift correction. Both IR beams are coupled to the main beam path via a dichroic mirror (ZT 785 SPXXR, Chroma Technology Corp., USA) and fed into the microscope to illuminate a region close, but not overlapping with the field of view used for MINFLUX. The reflected and backscattered light is split with an additional 50:50 beam splitter cube (BS014, Thorlabs GmbH, Germany) from the excitation IR beam and detected on a single CMOS camera (Zelux, Thorlabs $\mathrm{GmbH}$, Germany) at different positions of the chip.

Setup control. . The piezo stage (P733.3CD, Physik Instrumente (PI) GmbH \&Co. KG, Germany) translates the sample in all three dimensions with a resolution of $0.3 \mathrm{~nm}$ when running in closedloop mode. All components of the setup including the piezo stage are controlled digitally and integrated via a custom version of the PyFLUX project. Further details and source-code of this control software version are available at https://github.com/zaehringer-Jonas/pyflux 


\section{Supplementary Section 2. Drift correction}

Both the xy position using fiducial markers and the $\mathrm{z}$ position using the reflected IR beam are recorded in every experiment. The achieved fiducial tracking precision in xy is better than $1 \mathrm{~nm}$ and the $\mathrm{z}$ precision is better than $10 \mathrm{~nm}$ which is sufficient for our expected p-MINFLUX localization precision. An active drift correction is typically applied during the measurements by means of a feedback loop between the xyz signal and the piezo stage (Supplementary Figure S2A). Typically, the active drift correction achieves a stabilization better than $5 \mathrm{~nm}$ in xy and better than $10 \mathrm{~nm}$ in $\mathrm{z}$ (standard deviation). Any residual xyz drift can be corrected via post-processing using the tracking information. The xyz stabilization system can also be used to scan an emitter over the field of view. Fiducial tracking data from Figure $2 \mathrm{C}$ is shown in Supplementary Figure S2B

A

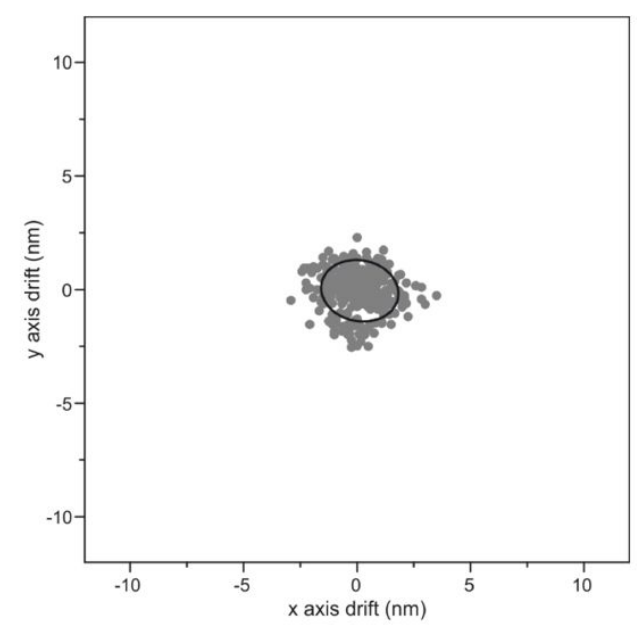

B

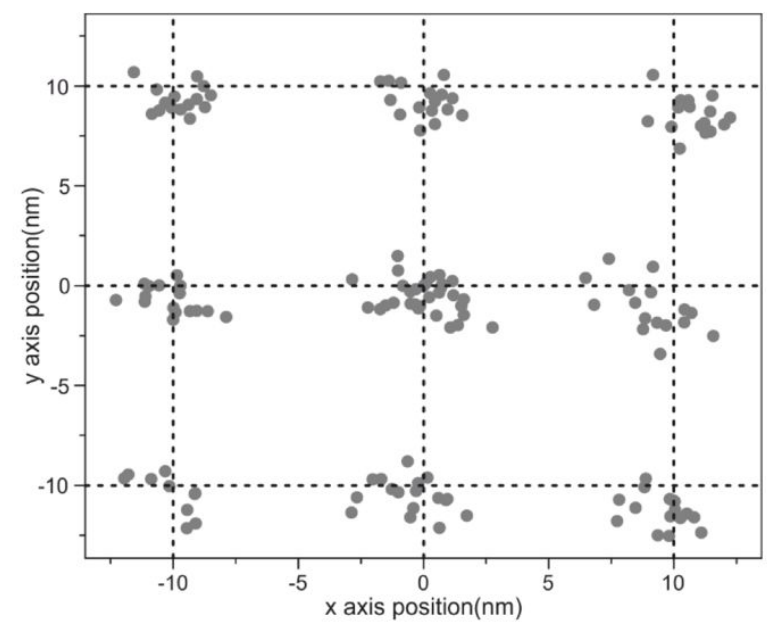

Supplementary Figure S2. A) Camera-based localization of fiducial markers during a 30 s point measurement and the covariance ellipse (black) showing a precision of $\sigma=1.5 \mathrm{~nm}$. B) Camerabased localization of fiducial markers during the measurement from Figure 2C. The piezo positioner is moved in a grid with a spacing of $10.0 \mathrm{~nm}$ in average, and an average precision of $\sigma=1.7 \mathrm{~nm}$. 


\section{Supplementary Section 3. DNA-origami structure folding}

The DNA origami structures were folded with a 10 -fold excess of staples to the scaffold in folding buffer $\left(20 \mathrm{mM} \mathrm{MgCl} 2 \times 6 \mathrm{H}_{2} \mathrm{O}, 40 \mathrm{mM}\right.$ Tris base, $20 \mathrm{mM}$ acetic acid, $1 \mathrm{mM}$ EDTA- $\left.\mathrm{Na}_{2} \times 2 \mathrm{H}_{2} \mathrm{O}\right)$ with a $16 \mathrm{~h}$ nonlinear folding ramp ${ }^{1}$.

Gel electrophoresis was used for purification. The $50 \mathrm{~mL}$ gel contains $1.5 \%$ agarose, $2 \mu \mathrm{L}$ peqGreen DNA stain and gel buffer $\left(12 \mathrm{mM} \mathrm{MgCl} 2 \times 6 \mathrm{H}_{2} \mathrm{O}, 40 \mathrm{mM}\right.$ Tris base, $20 \mathrm{mM}$ acetic acid, $1 \mathrm{mM}$ EDTA- $\mathrm{Na}_{2} \times 2 \mathrm{H}_{2} \mathrm{O}$ ). After adding $1 \times$ BlueJuice gel loading buffer to the DNA origami solution, the cooled gel runs for $2 \mathrm{~h}$ at $60 \mathrm{~V}$. Afterwards, the gel was cut, and the DNA origami solution was extracted via squeezing. The DNA origami concentration was measured with a NanoDrop 2000/2000c Spectrophotometer (ThermoFisher Scientific). 


\section{Supplementary Section 4. Sample preparation}

The coverslips were first washed using ultrapure water, then cleaned using an ozone cleaner (PSDUV4, Novascan Technologies, Inc., USA) and washed again using ultrapure water. For sample immobilization, the flow chamber was coated using biotin-labeled bovine serum albumin (1 $\mathrm{mg} / \mathrm{mL}$ Sigma-Aldrich Chemie $\mathrm{GmbH})$ and NeutrAvidin $(1 \mathrm{mg} / \mathrm{mL}$, Sigma-Aldrich Chemie $\mathrm{GmbH})$. In the flow chamber, the DNA origami structures (100 pM in $1 \mathrm{x}$ gel buffer) were immobilized due to the biotin-NeutrAvidin binding.

To suppress blinking and photobleaching, an oxidizing and reducing buffer system ${ }^{2}$ (1x TAE, 12 $\mathrm{mM} \mathrm{MgCl} 2,2 \mathrm{mM}$ trolox/troloxquinone, $1 \%(\mathrm{w} / \mathrm{v}) \mathrm{D}-(+)$ glucose) was used in combination with an oxygen scavenging system with a final concentration of $100 \mathrm{u} / \mathrm{ml}$ glucose oxidase and 2500 $\mathrm{u} / \mathrm{ml}$ catalase. To induce blinking for super-resolution imaging, $50 \mathrm{mM}$ beta-mercaptoethanol was added to the buffer (1x TAE, $12 \mathrm{mM} \mathrm{MgCl}_{2}, 2 \mathrm{mM}$ trolox/troloxquinone, 1\% (w/v) D-(+) glucose). $2 \mathrm{mM}$ cyclooctatetraene was added to increase the stability of the dye. 


\section{Supplementary Section 5. Determination of the excitation beam pattern and the point-spread functions}

To measure the exact center and shape of the four excitation beams (point-spread functions) an area of $400 \times 400 \mathrm{~nm}^{2}$ (pixel size: $2 \mathrm{~nm}$ ) around a fixed fluorescent nanoparticle (GATTAbead labeled with ATTO 542) was scanned with all the four excitation beams simultaneously. After separating the detected photons according to their arrival times (see Figure 1C in the main text) via the commercial software SymPhoTime64 (PicoQuant GmbH, Berlin, Germany), four images can be extracted (see Supplementary Figure S3, upper row) and fitted to a $4^{\text {th }}$ order polynomial in two dimensions. This fit result (Supplementary Figure S3, bottom row) is the basis for the position estimators as described in Supplementary Section 7.
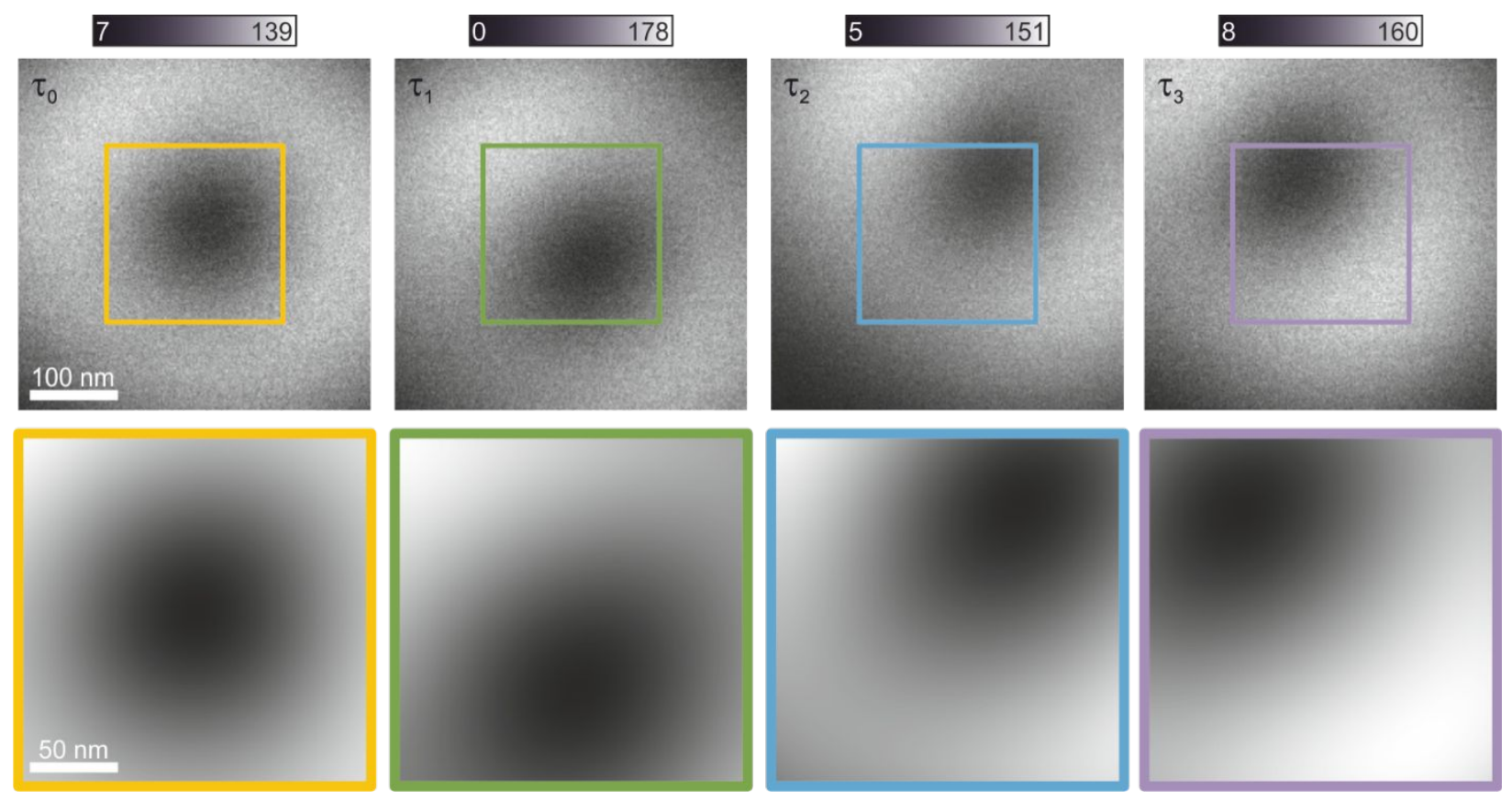

Supplementary Figure S3. Upper row: Simultaneous scan of one fluorescent ATTO542 GATTAbead with the four excitation beams; bottom row: Multi-polynomial fit of the central part 


\section{Supplementary Section 6. p-MINFLUX measurement}

At first, a large area $\left(10 \times 10 \mu \mathrm{m}^{2}\right)$ was scanned with the central donut of the excitation beam pattern (Figure S5). In this case, the sample consisted of a mixture of the structures described in Figure 4 of the main text. In the fluorescence lifetime image, the lifetimes of ATTO $542(\tau=3.2 \mathrm{~ns})$ and Cy3B ( $\tau=2.6 \mathrm{~ns})$ dyes are easily distinguished.
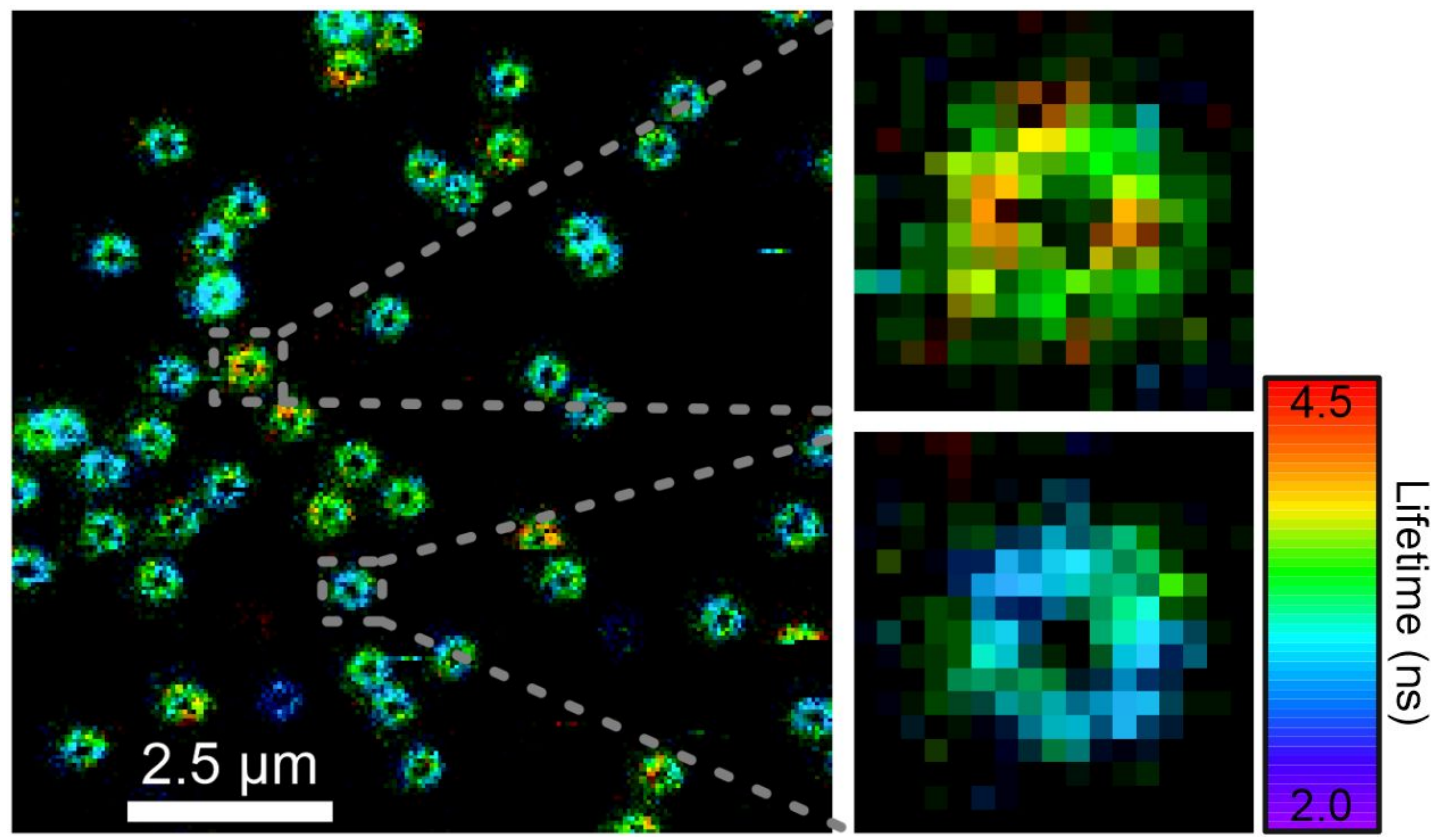

Supplementary Figure S4. 10x10 $\mu \mathrm{m}$ fluorescence lifetime scan image and zoom-in to two individual DNA-origami structures of a sample with a mixture of DNA origamis carrying ATTO 542 or $\mathrm{Cy} 3 \mathrm{~b}$ as described in Figure 4 of the main text.

Subsequently, the sample was moved in order to place a given DNA-origami at the center of the central donut. Then, the TCSPC measurement is started and the four laser foci were enabled. The measurements run until the molecule photobleaches. The TCSPC data contains the information about the emission intensity at each excitation beam and therefore can be used to obtain the position of the emitter as explained in the following section.

For the superresolution imaging experiments (Figure 3) molecular ON and OFF states have to be discerned. We used a time-binned trace and a suitable threshold to separate ON events from background signal (OFF events). The threshold was chosen well above the noise of the background signal to make sure no OFF states were considered as ON events (false positive) even at the expense of discarding possible low-count rate ON states (false negatives). We also discarded events with a very high photon count rate since they are likely to come from an event in which two 
molecules in the ON state are emitting at the same time. The trace displayed in Figure 3 is representative of the measurements. It was time-binned at $0.5 \mathrm{~ms}$, it had a background level of $\sim 10.2 \mathrm{kHz}$ with a noise of $\sim 2.4 \mathrm{kHz}$, and an average single-molecule ON state count rate of $\sim 80 \mathrm{kHz}$. A lower threshold of 25 counts, an upper threshold 80 counts, and a minimal ON-time length of $3 \mathrm{~ms}$ were used as threshold parameters. Then, every single-molecule emission event is analyzed separately and an $(x, y)$ position estimation is obtained as explained in Supplementary Section 7. 


\section{Supplementary Section 7. Position estimator and Cramér-Rao bound}

To compute the position estimators and to evaluate the theoretical maximum localization precision we followed the work by Balzarotti et al. ${ }^{3}$ We outline here the most important results used in this work.

\section{$\underline{\text { Position estimator }}$}

The likelihood function of the (p-)MINFLUX experiment for a given position of the single emitter $\vec{r}=(x, y)$ and $K$ expositions can be written as

$$
\mathcal{L}(\vec{r} \mid \vec{n})=\frac{N !}{\prod_{i=0}^{K-1} n_{i} !} \prod_{i=0}^{K-1} p_{i}(\vec{r})^{n_{i}}
$$

For all our experiments $K=4$. The multinomial parameters $p_{i}$ depend on the spatial coordinates and are defined as

$$
p_{i}(\vec{r})=\frac{S B R}{S B R+1} \frac{I_{i}(\vec{r})}{\sum_{j=0}^{K-1} I_{j}(\vec{r})}+\frac{1}{S B R+1} \frac{1}{K}
$$

where $I_{i}(\vec{r})$ are the intensity profiles of the excitation foci and SBR is the signal-to-background ratio of the experiment. To estimate the spatial position of the single emitter from the detected photons we used a Maximum Likelihood Estimator (MLE). Since $\mathcal{L}$ and $l=\ln (\mathcal{L})+$ const share the position of its maximum, we compute the log-likelihood function as

$$
l(\vec{r} \mid \bar{n})=\sum_{i=0}^{K-1} n_{i} \ln p_{i}(\vec{r})
$$

We note that in p-MINFLUX $n_{i}$ are the number of photons detected for each time window of detection, and that $p_{i}$ are calculated according to (S2) based on the experimental measurements of the arrangement of foci. The maximum of $l$ is found numerically and thus the MLE for $\vec{r}$ is 


$$
\hat{r}^{M L E}=\arg \max (l)
$$

All functions used to analyze the data arrays containing the arrival time of the photons, to construct $l$ and to find the MLE are fully implemented in Python and are open-source and available at https://github.com/lumasullo/p-minflux/blob/main/tools/tools_analysis.py and https://github.com/cibion-conicet/p-minflux/blob/main/tools/tools_analysis.py

Custom scripts using those functions were written to analyze every experiment. Those scripts are available upon request.

\section{Cramér-Rao bound}

To evaluate the theoretical lower bound in uncertainty we computed the Cramér-Rao bound for our experimental arrangement of excitation foci and also for a camera-based single-molecule localization approach. The Cramér-Rao bound expression for an arrangement of four beam expositions can be derived ${ }^{3}$ as the inverse of the Fisher information matrix which can, in turn, be derived from the Likelihood function of the experiment. For a two-dimensional (p-)MINFLUX experiment the Fisher information matrix is

$$
\mathcal{J}=N \sum_{i=0}^{K-1} \frac{1}{p_{i}}\left[\begin{array}{ll}
\left(\frac{\partial p_{i}}{\partial x}\right)^{2} & \frac{\partial p_{i} \partial p_{i}}{\partial x \partial y} \\
\frac{\partial p_{i} \partial p_{i}}{\partial y \partial x} & \left(\frac{\partial p_{i}}{\partial y}\right)^{2}
\end{array}\right]
$$

and hence the CRB matrix is defined as the inverse of $\mathcal{J}$ and poses a lower bound for the covariance of the estimator of the position

$$
\Sigma_{c o v}(\vec{r}) \geq \Sigma_{C R B}(\vec{r})=\mathfrak{J}^{-1} .
$$

The eigenvalues of $\Sigma_{C O V}$ and $\Sigma_{C R B}$ are denoted as $\sigma_{x}{ }^{2}$ and $\sigma_{y}{ }^{2}$. It is common practice to represent $\Sigma_{c o v}$ and $\Sigma_{C R B}$ as ellipses with the principal axes directions given by the eigenvectors of the matrices and the length of the axes given by $2 \sigma_{x}$ and $2 \sigma_{y}$ respectively. This representation is used 
in Figure 2 of the main text and Supplementary Figure S2, and is useful to illustrate the anisotropies in the MLE precision due to the geometry of the excitation beam pattern.

In order to evaluate the 2-D precision of the localizations with a scalar value, we use the same expression as used by Balzarotti et al. ${ }^{3}$, namely

$$
\sigma_{C R B}=\sqrt{\frac{1}{2} \operatorname{tr}\left(\Sigma_{C R B}\right)}
$$

Consequently, we chose to evaluate our experimental precision with an analog expression given by

$$
\sigma_{\text {exp }}=\sqrt{\frac{1}{2} \operatorname{tr}\left(\Sigma_{c o v}\right)}
$$

This definition of $\sigma$ is used for the calibration measurements in Figure 2 of the main text. For the $\sigma$ values reported in Figures 3 and 4, we used the $\sigma$ parameter of the Gaussian function that resulted from the fit of the $1 \mathrm{D}$ projections.

For the comparison with camera-based single-molecule localization techniques, we used the same expression for $\sigma_{C R B}$ but using the $p_{i}(x, y)$ given by

$$
\begin{gathered}
p_{i}(x, y)=\frac{1}{K+S B R_{C}}+\frac{S B R_{C}}{K+S B R_{C}} \cdot \frac{1}{4} \cdot\left(\operatorname{erf}\left(\frac{x_{i}+a / 2-x}{\sqrt{2} \sigma_{P S F}}\right)-\operatorname{erf}\left(\frac{x_{i}-a / 2-x}{\sqrt{2} \sigma_{P S F}}\right)\right) \\
\cdot\left(\operatorname{erf}\left(\frac{y_{i}+a / 2-y}{\sqrt{2} \sigma_{P S F}}\right)-\operatorname{erf}\left(\frac{y_{i}-a / 2-y}{\sqrt{2} \sigma_{P S F}}\right)\right)
\end{gathered}
$$

Where $a$ is the pixel size of the simulated camera and $\sigma_{P S F}$ the $\sigma$ of the simulated emission gaussian PSF (not to be confused with localization precision) and $K$ the number of pixels taken into account around the single-molecule image. The index $i$ indicates the pixel, while $(x, y)$ indicate the coordinate of the position of the emitter. For the simulations in Figure 2, we used $a=100 \mathrm{~nm}$, $K=81$ (9 99 pixels), and $\sigma_{P S F}=100 \mathrm{~nm}$

All the Python functions that we wrote to compute the CRB of both p-MINFLUX and camerabased SMLM to compare with our experimental data are open-source and can be found at 
https://github.com/lumasullo/p-minflux/blob/main/tools/tools simulations.py

and https://github.com/cibion-conicet/p-minflux/blob/main/tools/tools_simulations.py 


\section{Supplementary Section 8. p-MINFLUX simulations}

To estimate the overall performance of p-MINFLUX, as well as the influence of the different experimental parameters, we developed a software package with functions that simulate a complete p-MINFLUX experiment, including pulsed excitation with different beam patterns, fluorescence emission, TCSPC detection, and the position estimation of the emitter from the photon arrival times.

Briefly, in a first step doughnut-shaped foci (or foci with any other geometry) are created. Next, given a certain position of the emitter within the foci arrangement, an array of $\bar{n}=\left[n_{0}, n_{1}, n_{2}, n_{3}\right]$ photons is extracted from a multinomial distribution. Photon arrival times are generated based on predefined emission statistics; we used a single exponential distribution in agreement with the observed behavior of the fluorophores. The simulated photon arrival times are registered in both the fluorescence lifetime scale (i.e. from the laser excitation pulse; micro time) and in the absolute time scale of the simulation (i.e. from the beginning of the simulated experiment; macro time). Background photons with a uniform distribution of both macro and micro time are added in order to simulate different SBR situations.

At this point, the analysis is performed exactly in the same way as with the real data by calculating the MLE of the position of the emitter as explained in Supplementary Section 7. For each desired set of experimental parameters typically $M=1000$ simulations of p-MINFLUX were performed obtaining the full probability distribution of the estimator in a Monte-Carlo fashion. Typically, the mean error of the distribution was used as the localization quality metric.

$$
\varepsilon=\sqrt{\frac{1}{2 M} \sum_{i=1}^{M}\left(\left(x_{i}-x\right)^{2}+\left(y_{i}-y\right)^{2}\right)}
$$

where $M$ are the number of simulated results, $(x, y)$ indicate the ground truth position of the emitter and $\left(x_{i}, y_{i}\right)$ are the result of the i-th simulation. It should be noted that (S10) is very similar to equation (S7) but uses the ground truth position instead of the mean estimated position for the calculation of the trace of the covariance matrix thus accounting for the error due to any possible bias.

Functions used to simulate the experiments are open-source and can be found at https://github.com/lumasullo/p-minflux/blob/main/tools/tools simulations.py and https://github.com/cibion-conicet/p-minflux/blob/main/tools/tools_simulations.py 


\section{Supplementary Section 9. Effect of misalignment of the excitation beam pattern}

Unlike the MINFLUX implementation by Balzarotti et al. ${ }^{3}$, the excitation beam pattern (EBP) in p-MINFLUX is neither scanned during the measurement nor positioned with any active control or feedback loop. The alignment of the beams into the desired arrangement (usually an equilateral triangle with one beam in its center) is achieved by operating the kinematic mirrors mounts. In the experiments presented this was done manually and controlled through measurements as described in Supplementary Section 5. We were able to routinely align the EBP with 5-10 nm accuracy, in both set-ups (Supplementary Section 1).

We performed Monte-Carlo simulations to quantitatively analyze the influence of EBP misalignments on the attainable localization precision. Different emitter positions within the EPB were simulated and the resulting localization precisions were visualized in 2D maps. The EBP centers were displaced from their ideal positions by a radial offset $r$. During one simulation run, all four-excitation point spread functions (PSFs) constituting the EBP were off-set by the same value $r$.

For each emitter position and radial offset, we performed 1000 simulation runs. The angular direction of each PSF's displacement was randomly chosen and changed after 100 runs. This procedure of analyzing 10 random EBP for the same $r$ allowed us to find an averaged result which does not overestimate a particularly good or bad EBP. The simulations were carried out for $N=1000$ detected photons, $S B R=20$, and $L=100 \mathrm{~nm}$. In Supplementary Figure S5A, we show the exemplary simulation results for one random EBP with offsets of $r=0,10$ and $20 \mathrm{~nm}$, respectively.

To facilitate the evaluation of the simulation results, we computed $\sigma(0,0)$, the localization precision of an emitter placed at the center of the ideal EBP and $\langle\sigma\rangle_{F O V}$, the mean localization precision within the emitter position space of each 2D map. Figure S5B shows that the localization precision for an emitter at the EBP center decreases significantly for radial offsets $r>20 \mathrm{~nm}$. The mean localization precision, $\langle\sigma\rangle_{F O V}$, deteriorates by less than $0.5 \mathrm{~nm}$ for radial offsets smaller than $15 \mathrm{~nm}$.

Appreciating the experimental and computational findings, we conclude that the attainable localization precision in p-MINFLUX is robust against moderate misalignments of the EBP. Displacements of the PSFs' from their target positions by less than $15 \mathrm{~nm}$ do not lead to a significant decrease in localization precision. It must be emphasized that our analyses assumed PSFs which are measured and fitted with high precision and accuracy, as described in Supplementary Section 5. 
A

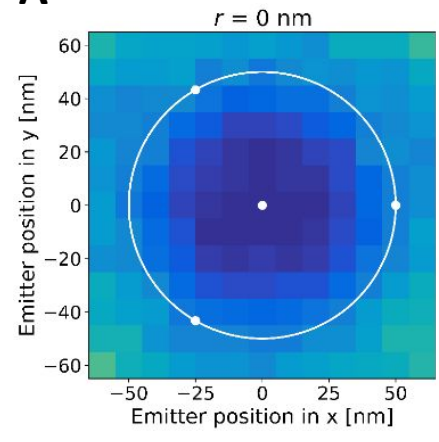

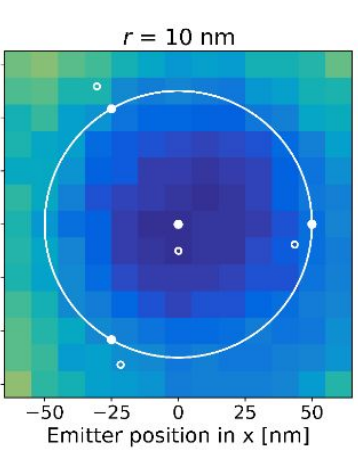

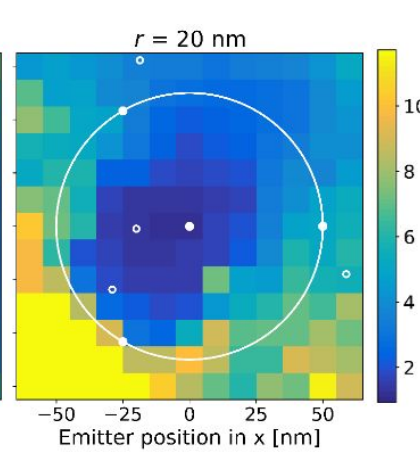

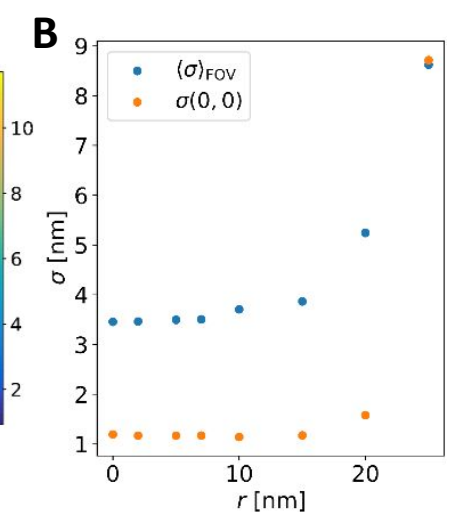

Supplementary Figure S5. Analysis of misalignments of the p-MINFLUX excitation beam pattern (EBP). A) Example 2D maps of the attainable localization precision for different emitter positions and radial EBP offsets $r=0,10$ and $20 \mathrm{~nm}$. The solid dots indicate the PSFs' center position of the ideal EBP whereas the small circles correspond to the misaligned EBP. B) The localization precision at the EBP center, $\sigma(0,0)$, and the mean over the emitter position space, $\langle\sigma\rangle_{F O V}$, is plotted for different radial offset values. The simulations were performed for $N=1000$ detected photons, $S B R=20$ and 10 randomly misaligned EBP for each $r$. 


\section{Supplementary Section 10. Photon arrival times and crosstalk between detection time windows}

As any other pulsed-interleaved technique, p-MINFLUX relies on the fact that minimal cross-talk exists between the arrival time-windows corresponding to each of the pulsed laser excitations. The probability of cross-talk can be calculated analytically taking into account the fluorescence lifetime of the molecule and the size of the detection time-windows.

The expected influence of the photon crosstalk on the p-MINFLUX localization precision and accuracy is however not straightforward. Thus we simulated p-MINFLUX experiments at different degrees of crosstalk. In Supplementary Figure S6A, we show simulated localization precision and accuracy for typical values of $N=1200, S B R=15, L=100 \mathrm{~nm}$ and fluorescence lifetimes ranging from 1.0 to $4.0 \mathrm{~ns}$ for detection time windows of $12.5 \mathrm{~ns}$. From our simulations, we conclude that effects on precision and accuracy are $<1 \mathrm{~nm}$ for fluorescence lifetimes up to $3.0 \mathrm{~ns}$ , which corresponds to roughly $1 / 4$ of the detection time window and $\sim 1 \%$ crosstalk. Measurements above this limit are still acceptable although a $\sim 2 \mathrm{~nm}$ bias is present and precision decreases slightly compared to the 1.0 ns (showing almost no crosstalk effect). In particular, we simulate the experimental situation of our super-resolution imaging experiments (Figure 3 of main text) and we found that despite the $\sim 5 \%$ crosstalk, p-MINFLUX is expected to fully resolve the DNA-origami structure (Supplementary Figure S6B). 
A

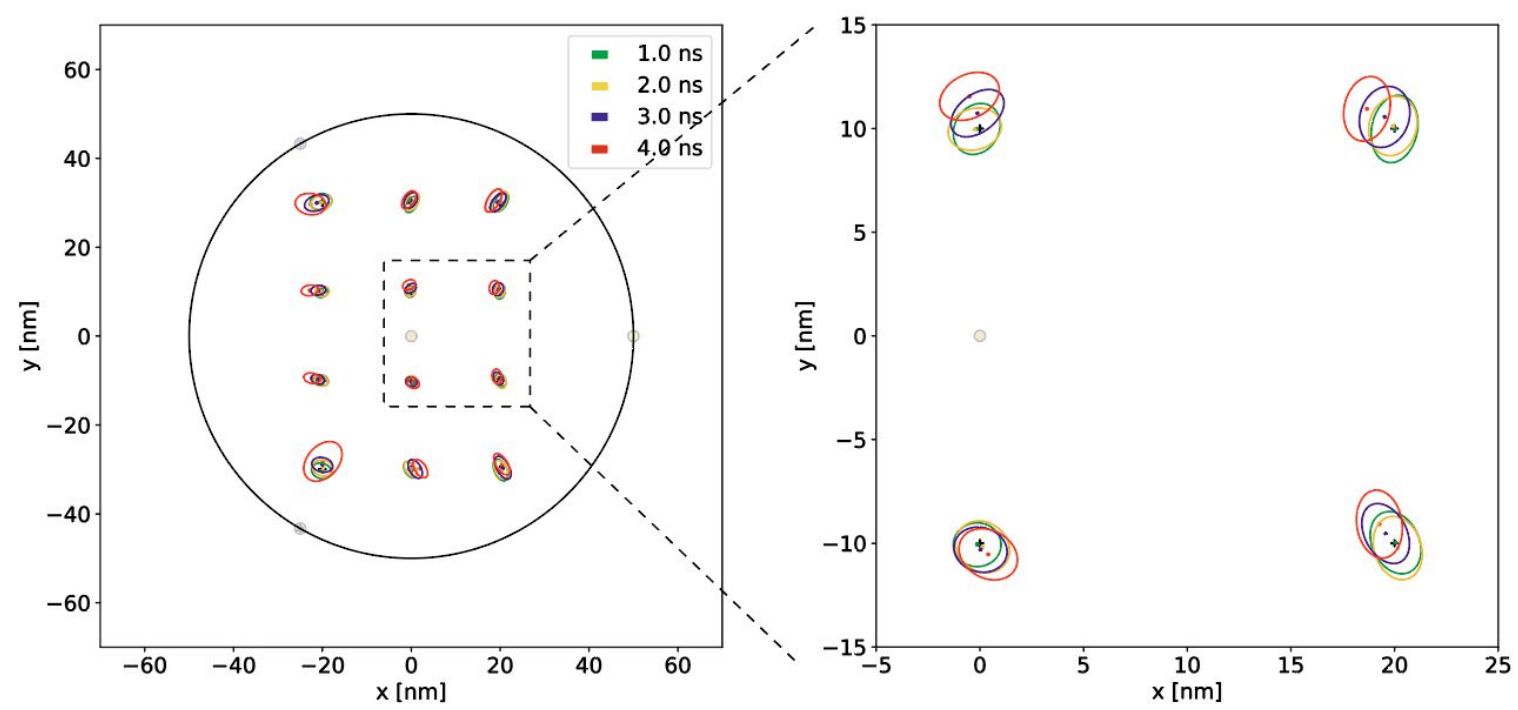

B

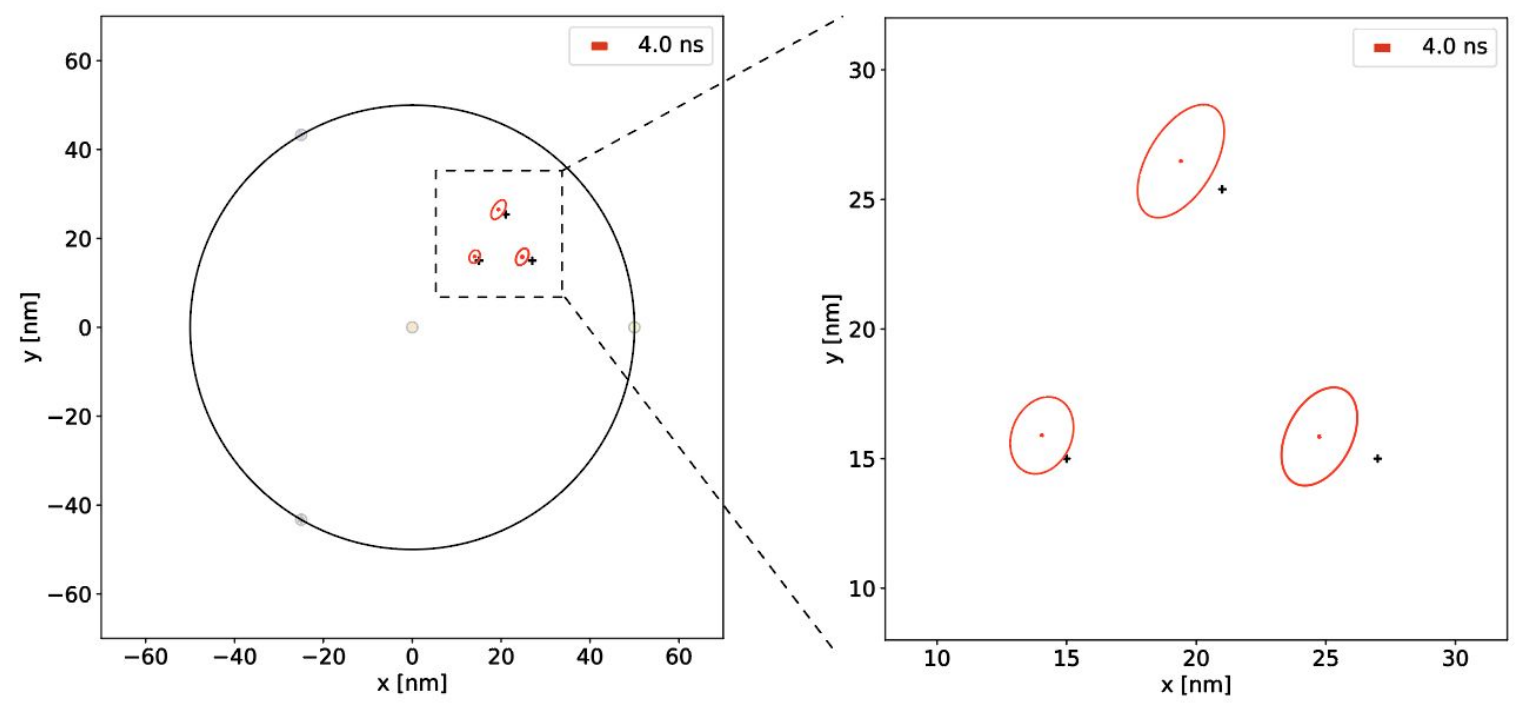

Supplementary Figure S6. A) Precision and accuracy of p-MINFLUX at different positions within the field of view for different fluorescence lifetimes. B) Simulation of a representative imaging experiment with a DNA-origami labeled with fluorophores with a fluorescence lifetime of $\sim 4.0 \mathrm{~ns}$ in analogy to the data presented in Figure 3 of the main text. Black crosses indicate the ground truth of the simulations.

We note that although in the Likelihood function (Equation S1) of the experiment we neglect crosstalk, the arrival times of the photons could be directly modeled, and hence the analysis 
optimized and very likely extend the scope of p-MINFLUX for larger crosstalk provided good SNR.

Finally, we note that since the crosstalk probability scales with the repetition rate of the laser, a lower repetition rate would solve the crosstalk and extend the range of possible fluorescence lifetimes. In particular, we demonstrated p-MINFLUX with a $20 \mathrm{MHz}$ pulsed laser, hence allowing to use of fluorophores and other single emitters with fluorescence (luminescence) lifetimes up to $\sim 4 \mathrm{~ns}$. A setup with a repetition rate of $10 \mathrm{MHz}$ would imply minimal changes to our current setup and extend the range of suitable fluorescence lifetimes up to $\sim 8 \mathrm{~ns}$ which cover most of the organic fluorophores typically used in fluorescence microscopy and spectroscopy. 


\section{Supplementary Section 11. Localization time traces simulation}

To estimate the expected distribution of binding times measured from finite, photon-limited localization traces we simulated the p-MINFLUX tracking experiment assuming the switching between positions of the DNA pointers (Figure 4) is stochastic and memory-less, and hence the transient binding times are distributed exponentially. We thus generated simulated p-MINFLUX localization traces with transient binding times at the two positions in the DNA-origami structure. We simulated the localizations to have a precision of $\sigma=3 \mathrm{~nm}$ and the distance between the two positions was set to $d=12 \mathrm{~nm}$. We generated traces with parameters (fluorescent lifetime, approximate binding time, number of transitions between positions) taken from our experimental data for both DNA-origami designs. Traces were then analyzed, and average binding times and fluorescent lifetimes were extracted using the same algorithms that we used to analyze the experimental data. 
Supplementary Table S1: Unmodified staples from the 5' to the 3' end for the DNA-origami structure used for the superresolution imaging experiments (Figure 3).

\begin{tabular}{|l|l|}
\hline Sequence (5' to $\mathbf{~}^{\prime}$ ) & length [nt] \\
\hline TAAGAGCAAATGTTTAGACTGGATAGGAAGCC & 32 \\
\hline AATAGTAAACACTATCATAACCCTCATTGTGA & 32 \\
\hline TCAAATATAACCTCCGGCTTAGGTAACAATTT & 32 \\
\hline GTACCGCAATTCTAAGAACGCGAGTATTATTT & 32 \\
\hline ATTATCATTCAATATAATCCTGACAATTAC & 30 \\
\hline AAATTAAGTTGACCATTAGATACTTTTGCG & 30 \\
\hline ATACATACCGAGGAAACGCAATAAGAAGCGCATTAGACGG & 40 \\
\hline AAAGTCACAAAATAAACAGCCAGCGTTTTA & 30 \\
\hline AAAGGCCGGAGACAGCTAGCTGATAAATTAATTTTTGT & 38 \\
\hline ACAAACGGAAAAGCCCCAAAAACACTGGAGCA & 32 \\
\hline TCTTCGCTGCACCGCTTCTGGTGCGGCCTTCC & 32 \\
\hline GACAAAAGGTAAAGTAATCGCCATATTTAACAAAACTTTT & 40 \\
\hline GATTTAGTCAATAAAGCCTCAGAGAACCCTCA & 32 \\
\hline TATATTTTGTCATTGCCTGAGAGTGGAAGATTGTATAAGC & 40 \\
\hline GCTTTCCGATTACGCCAGCTGGCGGCTGTTTC & 32 \\
\hline CACCAGAAAGGTTGAGGCAGGTCATGAAAG & 30 \\
\hline CGCGCAGATTACCTTTTTTAATGGGAGAGACT & 32 \\
\hline ACAACATGCCAACGCTCAACAGTCTTCTGA & 30 \\
\hline GTTTATCAATATGCGTTATACAAACCGACCGTGTGATAAA & 30 \\
\hline AACGCAAAGATAGCCGAACAAACCCTGAAC & 32 \\
\hline CTTTTACAAAATCGTCGCTATTAGCGATAG & 30 \\
\hline CAACTGTTGCGCCATTCGCCATTCAAACATCA & 32 \\
\hline CAGCGAAACTTGCTTTCGAGGTGTTGCTAA & 30 \\
\hline AAGGCCGCTGATACCGATAGTTGCGACGTTAG & 32 \\
\hline
\end{tabular}




\begin{tabular}{|c|c|}
\hline AGGCTCCAGAGGCTTTGAGGACACGGGTAA & 30 \\
\hline TTAGGATTGGCTGAGACTCCTCAATAACCGAT & 32 \\
\hline GATGGCTTATCAAAAAGATTAAGAGCGTCC & 30 \\
\hline TAAATCGGGATTCCCAATTCTGCGATATAATG & 32 \\
\hline AGGCAAAGGGAAGGGCGATCGGCAATTCCA & 30 \\
\hline TTTCGGAAGTGCCGTCGAGAGGGTGAGTTTCG & 32 \\
\hline TCACCAGTACAAACTACAACGCCTAGTACCAG & 32 \\
\hline AACAAGAGGGATAAAAATTTTTAGCATAAAGC & 32 \\
\hline AAATCACCTTCCAGTAAGCGTCAGTAATAA & 30 \\
\hline CAGCAAAAGGAAACGTCACCAATGAGCCGC & 30 \\
\hline TGTAGCCATTAAAATTCGCATTAAATGCCGGA & 32 \\
\hline TATTAAGAAGCGGGGTTTTTGCTCGTAGCAT & 30 \\
\hline TACGTTAAAGTAATCTTGACAAGAACCGAACT & 32 \\
\hline GCAAGGCCTCACCAGTAGCACCATGGGCTTGA & 32 \\
\hline TGCATCTTTCCCAGTCACGACGGCCTGCAG & 30 \\
\hline AATTGAGAATTCTGTCCAGACGACTAAACCAA & 32 \\
\hline GCCATCAAGCTCATTTTTTTAACCACAAATCCA & 32 \\
\hline TAAATGAATTTTTCTGTATGGGATTAATTTCTT & 32 \\
\hline ATGCAGATACATAACGGGAATCGTCATAAATAAAGCAAAG & 40 \\
\hline AATACGTTTGAAAGAGGACAGACTGACCTT & 30 \\
\hline TTATACCACCAAATCAACGTAACGAACGAG & 30 \\
\hline CATGTAATAGAATATAAAGTACCAAGCCGT & 30 \\
\hline TATAACTAACAAAGAACGCGAGAACGCCAA & 30 \\
\hline ATCGCAAGTATGTAAATGCTGATGATAGGAAC & 32 \\
\hline CATAAATCTTTGAATACCAAGTGTTAGAAC & 30 \\
\hline GCGAAAAATCCCTTATAAATCAAGCCGGCG & 30 \\
\hline TAAATCATATAACCTGTTTAGCTAACCTTTAA & 32 \\
\hline CTTTAGGGCCTGCAACAGTGCCAATACGTG & 30 \\
\hline
\end{tabular}




\begin{tabular}{|c|c|}
\hline CGAAAGACTTTGATAAGAGGTCATATTTCGCA & 32 \\
\hline AGCAAGCGTAGGGTTGAGTGTTGTAGGGAGCC & 32 \\
\hline TCAAGTTTCATTAAAGGTGAATATAAAAGA & 30 \\
\hline TCATTCAGATGCGATTTTAAGAACAGGCATAG & 32 \\
\hline CTACCATAGTTTGAGTAACATTTAAAATAT & 30 \\
\hline ATCCCAATGAGAATTAACTGAACAGTTACCAG & 32 \\
\hline ACCTTGCTTGGTCAGTTGGCAAAGAGCGGA & 30 \\
\hline CTGTGTGATTGCGTTGCGCTCACTAGAGTTGC & 32 \\
\hline GCCGTCAAAAAACAGAGGTGAGGCCTATTAGT & 32 \\
\hline GTATAGCAAACAGTTAATGCCCAATCCTCA & 30 \\
\hline AGACGACAAAGAAGTTTTGCCATAATTCGAGCTTCAA & 37 \\
\hline ACCCTTCTGACCTGAAAGCGTAAGACGCTGAG & 32 \\
\hline ATATTTTTGGCTTTCATCAACATTATCCAGCCA & 32 \\
\hline ATATTCGGAACCATCGCCCACGCAGAGAAGGA & 32 \\
\hline TTCCAGTCGTAATCATGGTCATAAAAGGGG & 30 \\
\hline CGGATTGCAGAGCTTAATTGCTGAAACGAGTA & 32 \\
\hline AAAGCACTAAATCGGAACCCTAATCCAGTT & 30 \\
\hline GACCTGCTCTTTGACCCCCAGCGAGGGAGTTA & 32 \\
\hline GTTTATTTTGTCACAATCTTACCGAAGCCCTTTAATATCA & 40 \\
\hline TAATCAGCGGATTGACCGTAATCGTAACCG & 30 \\
\hline GCGAGTAAAAATATTTAAATTGTTACAAAG & 30 \\
\hline CTTAGATTTAAGGCGTTAAATAAAGCCTGT & 30 \\
\hline CCAGGGTTGCCAGTTTGAGGGGACCCGTGGGA & 32 \\
\hline TAGGTAAACTATTTTTGAGAGATCAAACGTTA & 32 \\
\hline CGATAGCATTGAGCCATTTGGGAACGTAGAAA & 32 \\
\hline GCGCAGACAAGAGGCAAAAGAATCCCTCAG & 30 \\
\hline CCTGATTGCAATATATGTGAGTGATCAATAGT & 32 \\
\hline GCCCGAGAGTCCACGCTGGTTTGCAGCTAACT & 32 \\
\hline
\end{tabular}




\begin{tabular}{|c|c|}
\hline TCATCGCCAACAAAGTACAACGGACGCCAGCA & 32 \\
\hline CTGTAGCTTGACTATTATAGTCAGTTCATTGA & 32 \\
\hline GAATTTATTTAATGGTTTGAAATATTCTTACC & 32 \\
\hline CCTAAATCAAAATCATAGGTCTAAACAGTA & 30 \\
\hline AGCGCGATGATAAATTGTGTCGTGACGAGA & 30 \\
\hline GATGTGCTTCAGGAAGATCGCACAATGTGA & 30 \\
\hline GCCTCCCTCAGAATGGAAAGCGCAGTAACAGT & 32 \\
\hline TTTATCAGGACAGCATCGGAACGACACCAACCTAAAACGA & 40 \\
\hline CACAACAGGTGCCTAATGAGTGCCCAGCAG & 30 \\
\hline CTGAGCAAAAATTAATTACATTTTGGGTTA & 30 \\
\hline TGACAACTCGCTGAGGCTTGCATTATACCA & 30 \\
\hline GCGAACCTCCAAGAACGGGTATGACAATAA & 30 \\
\hline TGAAAGGAGCAAATGAAAAATCTAGAGATAGA & 32 \\
\hline GCACAGACAATATTTTTGAATGGGGTCAGTA & 31 \\
\hline TCACCGACGCACCGTAATCAGTAGCAGAACCG & 32 \\
\hline GCCCTTCAGAGTCCACTATTAAAGGGTGCCGT & 32 \\
\hline CCACCCTCTATTCACAAACAAATACCTGCCTA & 32 \\
\hline ATTTTAAAATCAAAATTATTTGCACGGATTCG & 32 \\
\hline GTAATAAGTTAGGCAGAGGCATTTATGATATT & 32 \\
\hline TTAACACCAGCACTAACAACTAATCGTTATTA & 32 \\
\hline TTTTATTTAAGCAAATCAGATATTTTTTTGT & 30 \\
\hline AACACCAAATTTCAACTTTAATCGTTTACC & 30 \\
\hline GATGGTTTGAACGAGTAGTAAATTTACCATTA & 32 \\
\hline CTCGTATTAGAAATTGCGTAGATACAGTAC & 30 \\
\hline ATCCCCCTATACCACATTCAACTAGAAAAATC & 32 \\
\hline CTTATCATTCCCGACTTGCGGGAGCCTAATTT & 32 \\
\hline GCGGAACATCTGAATAATGGAAGGTACAAAAT & 32 \\
\hline GACCAACTAATGCCACTACGAAGGGGGTAGCA & 32 \\
\hline
\end{tabular}




\begin{tabular}{|c|c|}
\hline ACGGCTACAAAAGGAGCCTTTAATGTGAGAAT & 32 \\
\hline TGGAACAACCGCCTGGCCCTGAGGCCCGCT & 30 \\
\hline GCGGATAACCTATTATTCTGAAACAGACGATT & 32 \\
\hline CCCGATTTAGAGCTTGACGGGGAAAAAGAATA & 32 \\
\hline TTAAAGCCAGAGCCGCCACCCTCGACAGAA & 30 \\
\hline CAGAAGATTAGATAATACATTTGTCGACAA & 30 \\
\hline AGAAAACAAAGAAGATGATGAAACAGGCTGCG & 32 \\
\hline CGTAAAACAGAAATAAAAATCCTTTGCCCGAAAGATTAGA & 40 \\
\hline ACCGATTGTCGGCATTTTCGGTCATAATCA & 30 \\
\hline AATAGCTATCAATAGAAAATTCAACATTCA & 30 \\
\hline AAACAGCTTTTTGCGGGATCGTCAACACTAAA & 32 \\
\hline ATTATACTAAGAAACCACCAGAAGTCAACAGT & 32 \\
\hline TTGACAGGCCACCACCAGAGCCGCGATTTGTA & 32 \\
\hline TACCGAGCTCGAATTCGGGAAACCTGTCGTGCAGCTGATT & 40 \\
\hline GCCAGTTAGAGGGTAATTGAGCGCTTTAAGAA & 32 \\
\hline AGCCAGCAATTGAGGAAGGTTATCATCATTTT & 32 \\
\hline CATTTGAAGGCGAATTATTCATTTTTGTTTGG & 32 \\
\hline ACACTCATCCATGTTACTTAGCCGAAAGCTGC & 32 \\
\hline ACCTTTTTTATTTTAATTAAATTTCATAGGGCTT & 32 \\
\hline GCTATCAGAAATGCAATGCCTGAATTAGCA & 30 \\
\hline AAGTAAGCAGACACCACGGAATAATATTGACG & 32 \\
\hline CAGGAGGTGGGGTCAGTGCCTTGAGTCTCTGAATTTACCG & 40 \\
\hline TTAATGAACTAGAGGATCCCCGGGGGGTAACG & 32 \\
\hline AAGGAAACATAAAGGTGGCAACATTATCACCG & 32 \\
\hline CATCAAGTAAAACGAACTAACGAGTTGAGA & 30 \\
\hline AATGGTCAACAGGCAAGGCAAAGAGTAATGTG & 32 \\
\hline CAACCGTTTCAAATCACCATCAATTCGAGCCA & 32 \\
\hline GGCCTTGAAGAGCCACCACCCTCAGAAACCAT & 32 \\
\hline
\end{tabular}




\begin{tabular}{|c|c|}
\hline TAAATCAAAATAATTCGCGTCTCGGAAACC & 30 \\
\hline GAGGGTAGGATTCAAAAGGGTGAGACATCCAA & 32 \\
\hline TTATTACGAAGAACTGGCATGATTGCGAGAGG & 32 \\
\hline ATACCCAACAGTATGTTAGCAAATTAGAGC & 30 \\
\hline TTAACGTCTAACATAAAAACAGGTAACGGA & 30 \\
\hline AGTATAAAGTTCAGCTAATGCAGATGTCTTTC & 32 \\
\hline CACATTAAAATTGTTATCCGCTCATGCGGGCC & 32 \\
\hline CTTTAATGCGCGAACTGATAGCCCCACCAG & 30 \\
\hline GAAATTATTGCCTTTAGCGTCAGACCGGAACC & 32 \\
\hline AACGCAAAATCGATGAACGGTACCGGTTGA & 30 \\
\hline ACAACTTTCAACAGTTTCAGCGGATGTATCGG & 32 \\
\hline GCCTTAAACCAATCAATAATCGGCACGCGCCT & 32 \\
\hline TCCACAGACAGCCCTCATAGTTAGCGTAACGA & 32 \\
\hline GCCCGTATCCGGAATAGGTGTATCAGCCCAAT & 32 \\
\hline TCAATATCGAACCTCAAATATCAATTCCGAAA & 32 \\
\hline GTCGACTTCGGCCAACGCGCGGGGTTTTTC & 30 \\
\hline GCAATTCACATATTCCTGATTATCAAAGTGTA & 32 \\
\hline CTCCAACGCAGTGAGACGGGCAACCAGCTGCA & 32 \\
\hline TGTAGAAATCAAGATTAGTTGCTCTTACCA & 30 \\
\hline TTCTACTACGCGAGCTGAAAAGGTTACCGCGC & 32 \\
\hline CAAATCAAGTTTTTTGGGGTCGAAACGTGGA & 31 \\
\hline CCAATAGCTCATCGTAGGAATCATGGCATCAA & 32 \\
\hline TTAGTATCACAATAGATAAGTCCACGAGCA & 30 \\
\hline AGGAACCCATGTACCGTAACACTTGATATAA & 31 \\
\hline GAGAGATAGAGCGTCTTTCCAGAGGTTTTGAA & 32 \\
\hline TCGGCAAATCCTGTTTGATGGTGGACCCTCAA & 32 \\
\hline AAGCCTGGTACGAGCCGGAAGCATAGATGATG & 32 \\
\hline AGAAAGGAACAACTAAAGGAATTCAAAAAAA & 31 \\
\hline
\end{tabular}




\begin{tabular}{|l|l|}
\hline ACGCTAACACCCACAAGAATTGAAAATAGC & 30 \\
\hline CCACCCTCATTTTCAGGGATAGCAACCGTACT & 32 \\
\hline TTTAGGACAAATGCTTTAAACAATCAGGTC & 30 \\
\hline AGAGAGAAAAAAATGAAAATAGCAAGCAAACT & 32 \\
\hline AACGTGGCGAGAAAGGAAGGGAAACCAGTAA & 31 \\
\hline CCAACAGGAGCGAACCAGACCGGAGCCTTTAC & 32 \\
\hline AACAGTTTTGTACCAAAAACATTTTATTTC & 30 \\
\hline TTTACCCCAACATGTTTTAAATTTCCATAT & 30 \\
\hline TAAAAGGGACATTCTGGCCAACAAAGCATC & 30 \\
\hline TTTTCACTCAAAGGGCGAAAACCATCACC & 30 \\
\hline TCTAAAGTTTTGTCGTCTTTCCAGCCGACAA & 31 \\
\hline GTTTTAACTTAGTACCGCCACCCAGAGCCA & 30 \\
\hline
\end{tabular}


Supplementary Table S2. Modified and extended staples from the 5' to the 3' end for the DNAorigami structure used for the superresolution imaging experiments (Figure 3).

\begin{tabular}{|c|c|c|}
\hline Sequence $\left(5^{\prime}\right.$ to $\left.3^{\prime}\right)$ & Length [nt] & Function \\
\hline $\begin{array}{l}\text { Biotin- } \\
\text { GAGAAGAGATAACCTTGCTTCTGTTCGGGAGAAACAATAA }\end{array}$ & 40 & Biotin for immobilization \\
\hline $\begin{array}{l}\text { Biotin- } \\
\text { ATAAGGGAACCGGATATTCATTACGTCAGGACGTTGGGAA }\end{array}$ & 40 & Biotin for immobilization \\
\hline $\begin{array}{l}\text { Biotin- } \\
\text { AGCCACCACTGTAGCGCGTTTTCAAGGGAGGGAAGGTAAA }\end{array}$ & 40 & Biotin for immobilization \\
\hline $\begin{array}{l}\text { Biotin- } \\
\text { CGGATTCTGACGACAGTATCGGCCGCAAGGCGATTAAGTT }\end{array}$ & 40 & Biotin for immobilization \\
\hline $\begin{array}{l}\text { Biotin- } \\
\text { GAAACGATAGAAGGCTTATCCGGTCTCATCGAGAACAAGC }\end{array}$ & 40 & Biotin for immobilization \\
\hline $\begin{array}{l}\text { Biotin- } \\
\text { TAGAGAGTTATTTTCATTTGGGGATAGTAGTAGCATTA }\end{array}$ & 40 & Biotin for immobilization \\
\hline $\begin{array}{l}\text { TTCCTCTACCACCTACATCACAA } \\
\text { ATTACCTTTGAATAAGGCTTGCCCAAATCCGC }\end{array}$ & 56 & $\begin{array}{l}\text { External labeling site of Atto } \\
532 \text { for super resolution } \\
\text { imaging }\end{array}$ \\
\hline $\begin{array}{l}\text { TTCСTCTACСАССТАСАTCACAA } \\
\text { TTGCTCCTTTCAAATATCGCGTTTGAGGGGGT }\end{array}$ & 56 & $\begin{array}{l}\text { External labeling site of Atto } \\
532 \text { for super resolution } \\
\text { imaging }\end{array}$ \\
\hline $\begin{array}{l}\text { TTCCTCTACCACCTACATCACAA } \\
\text { AATACTGCCCAAAAGGAATTACGTGGCTCA }\end{array}$ & 54 & $\begin{array}{l}\text { External labeling site of Atto } \\
532 \text { for super resolution } \\
\text { imaging }\end{array}$ \\
\hline $\begin{array}{l}\text { TTCCTCTACCACCTACATCACAA } \\
\text { CTTTTGCAGATAAAAACCAAAATAAAGACTCC }\end{array}$ & 56 & $\begin{array}{l}\text { External labeling site of Atto } \\
532 \text { for super resolution } \\
\text { imaging }\end{array}$ \\
\hline Atto532-TTTGTGATGTAGGTGGTAGAGGAA & 25 & $\begin{array}{l}\text { Atto } 532 \text { external labeling } \\
\text { strand for super resolution } \\
\text { imaging }\end{array}$ \\
\hline GATGGCTTATCAAAA-Atto532-GATTAAGAGCGTCC & 30 & $\begin{array}{l}\text { Internal Atto } 532 \text { for } \\
\text { evaluation of the } \\
\text { localization performance }\end{array}$ \\
\hline
\end{tabular}


Supplementary Table S3: Unmodified staples from the 5' to the 3' end for the DNA-origami structure used for the tracking experiments (Figure 4).

\begin{tabular}{|l|c|}
\hline Sequence (5' to $\mathbf{3}^{\prime}$ ) & Length [nt] \\
\hline CAATTCATATAGATAATAAATCCTTTGCCCG & 31 \\
\hline ATGAGTGACCTGTGCAGTTTCTGCCAGCACG & 31 \\
\hline GGAACCCAAAACTACAAACAGTTTCAGCG & 29 \\
\hline GTTTTCCCGTAGATGGCAGGAAGATCGCACT & 31 \\
\hline TTTTTTAATGCACGTACAAGTTACCCATTCAG & 32 \\
\hline CCCGCCGCGCTTAATGAAAGCCGGCGAACGTG & 32 \\
\hline AAATCCCGTAAAAAAACGTTTTTTGGACTTGT & 32 \\
\hline AGGAAACCGAGGACGTAGAAAAAGTACCG & 29 \\
\hline TATCATTTTGCGGAACATCCTGATATAAAGAA & 32 \\
\hline CTTAATTGAGACCGGAACAGGTCAGGATTAGAGGTGGCA & 40 \\
\hline TTTTTTTTTTTTAAAACTAG & 20 \\
\hline GCCGATTAAGGAAGGGCGCGTAACCACCACA & 31 \\
\hline ATGGCTACAATCAACTGAGAGCCAGCAGCAAATGAAAAACGAACCTAATGCGCTTGGCAGA & 61 \\
\hline GTCCACTAAACGCGCGGACGGGCAACAGCTG & 31 \\
\hline TGCGGCCAGAATGCGGTTTT & 20 \\
\hline TTTTATTGGGCTTGAGATGGCCAGAACGATT & 31 \\
\hline ATTGCCCTTCACCGCCCCAGCTGCTTGCGTTG & 32 \\
\hline TGATGTTACTTAGCCGGAAAAGACAGCACTACGAA & 35 \\
\hline TTTTCTTTACAAACAATTCG & 20 \\
\hline TATTTTGTTAAAATTCGGGTATATATCAAAAC & 32 \\
\hline TAATAGTATTCTCCGTGCATTAAATTTTTGTT & 29 \\
\hline TGTTGCCCTGCGGCTGATCAGATGCAGTGTCA & 32 \\
\hline TTTTTTGCATCAAAAGCCTGAGTAATTTT & 32 \\
\hline TTTTTTGAGGGGACGACGAC & 32 \\
\hline
\end{tabular}




\begin{tabular}{|c|c|}
\hline TGATTGCTTTGAATACAAACAGAATGTTTGGA & 32 \\
\hline ACATAGCGATAGCTTATTTTT & 20 \\
\hline TTTTCCCTTACACTGGTTGC & 20 \\
\hline CGGCCTCGTTAGAATCTTTTT & 20 \\
\hline TTACCCATAACCCTCGAAATACAATGTTTAAACAGGG & 37 \\
\hline TTTTTTAGGAATACCACAGTAGTAATTTT & 29 \\
\hline TGTAATCTTGACAAGAACCGAAC & 23 \\
\hline CTTCTGACCTAAATTTGCAGAGGCCAGAACGCAATTTACG & 40 \\
\hline AGATGAAGGGTAAAGTTTTT & 20 \\
\hline CACAGACATTTCAGGGATCTCCAAAAAAAAGGTTCTTAAAGCCGCTTT & 48 \\
\hline TCTTTAGGCTGAATAATGCTCATTAGTAACAT & 32 \\
\hline CAAAGGGCCTGTCGTGTGGCCCTGAGAGAGTT & 32 \\
\hline GCAGAGGCGAATTATTTTTTCATTTGCTATTAA & 32 \\
\hline TTTCGACTTGATCGAGAGGGTTGATATAAGTATTTT & 36 \\
\hline TTTTCATATAAAAGAAAGCCGAACATTTT & 29 \\
\hline TTTTAAACATCAAGAAAAAA & 20 \\
\hline CAAAAGAATAAAATACCCAGCGATTATACCAAGCGCGAA & 39 \\
\hline GAGCCGATATAACAACAACCATCGCCCTTTTTTTT & 34 \\
\hline TTTTTTCCTGATTATCACGT & 20 \\
\hline CCGAATCTAAAGCATCTTTT & 20 \\
\hline TTTTGCTAATATCAGAGAGATAACCCCGCCACCGCG & 36 \\
\hline TGTACTGGTAATAAGTTCAGTGCC & 24 \\
\hline TTTTCGCAAATGGTCAATAAACCATTAGATGC & 32 \\
\hline ATCAAAAAGTCATAAAACGGAACAACATTATCAACTTTAGTAGAT & 45 \\
\hline GGCTTAGGTTGGGTTAAGCTAATGATTTTCGA & 32 \\
\hline AACGTTATTAATTTTACAACTAATCAGTTGGC & 32 \\
\hline TGCGAATAATAATCGACAATGTTCGGTCG & 29 \\
\hline CTGCAACAGTGCCACGTATCTGGTAGATTAGA & 32 \\
\hline
\end{tabular}




\begin{tabular}{|c|c|}
\hline CCAGCCAGCTTTCCGGGTAATGGGGTAACAAC & 32 \\
\hline CCGTGCATCTGCCAGTTTTT & 20 \\
\hline TTTTCCCGACTTACAAAATAAACAGTTTT & 29 \\
\hline GAAAGGAGCGGGCGCTAGGTTTT & 23 \\
\hline CATTGCCTGAGAGTCTTTATGACCATAAATCATTTCATTT & 40 \\
\hline TTTTGGCGCATAGGCTGGCTAACGGTGTTAAATTGT & 36 \\
\hline CTCCAATCGTCTGAAATTTT & 20 \\
\hline CCCCCTGCGCCCGCTTTAGCTGTTTCCTGTGT & 32 \\
\hline GGAGCCTTCACCCTCAGAGCCACC & 24 \\
\hline TTTTTTGTTCCAGTTTGGAACAAGA & 25 \\
\hline TTAATTAAACCATACATACATAAAGGTGGCAATTTT & 36 \\
\hline TTTTTATCATCGCCTGAACAGACCATTTT & 29 \\
\hline TCTTACCATAAAGCCATAATTTAGAATGGTTTAGGGTAGC & 40 \\
\hline TTTTATTGCTGAATATAATACATTTTTTTT & 29 \\
\hline AAATCAACACGTGGCATCAGTATTCTCAATCC & 32 \\
\hline AGAACGTTAACGGCGTAATGGGTAAAGGTTTCTTTGCGTCGGTGGTGCTGGTCTTGCCGTT & 61 \\
\hline GCCTAATTATCATATGATAAGAGATTTAGTTAATTTCAT & 39 \\
\hline TTTTCCATATTATTTATCCCAATCCAAAGTCAGAGA & 36 \\
\hline ACCCTCATGCCCTCATTTTCTGTATGGGATTTAGTTAAAGCAGCTTGA & 48 \\
\hline GTCGAAATCCGCGACCTGCTCCACCAACTTTTAAGCATTC & 39 \\
\hline CCGGAACCGCAAGAAAGCAATAGCTATCTTACTCACAATCCGATTGAG & 48 \\
\hline AACGTCAATAGACGGGGAATACCCAAAAGAACAAGACTCCGTTTTTAT & 48 \\
\hline GCAGTTGGGCGGTTGTCCAGTTATGGAAGGAG & 32 \\
\hline CCGTCGGAGTAGCATTCAAAAACAGGAAGATT & 32 \\
\hline TTTTTTATCACCGTCACAGCGTCAGTTTT & 29 \\
\hline CCAACATGACGCTCAATGCCGGAGGAAATACC & 32 \\
\hline TACAGGCATTAAATTAACCAATAGGAACGCCATCAAAGTCAATCAGAATTAGCCTAAATCG & 61 \\
\hline AGTTGATTAGCTGAAAAGAGTACCTTTAATTGTTAATTCGGACCATAA & 48 \\
\hline
\end{tabular}




\begin{tabular}{|c|c|}
\hline AGCGAACCAGAAGCCTGGAGAATCACAAAGGCTATCAGGT & 40 \\
\hline TTTTGAACAACTAAAGGAACACTGATTTT & 29 \\
\hline CGGGAAACGAAAAACCTGATGGTGGTTCCGAA & 32 \\
\hline CGTTGAAAATAGCAAGCCCAATA & 23 \\
\hline GACAGATGGACCTTCATCAAGAGCCCTGAC & 30 \\
\hline GCGAGAAAAGGGATGACGAGCACGTATAACGTGCTTTTCACGCTGAAGAAAGC & 53 \\
\hline TCGATAGCAGCACCGTAAAATCACGTTTTGCT & 32 \\
\hline TTTTCCCTCAGAGCCACCACCCTCAGAAAGCGCTTA & 36 \\
\hline ATCGGCAAAATCCCTTACGTGGACTCCAACGT & 32 \\
\hline TTCAAATTTTTAGAAAAAACAGGAGCAAACAAGAGAATCGATGAAGGGTGAGATATTTTA & 60 \\
\hline TTTTTAGCCCGGAATAGCCTATTTCTTTT & 29 \\
\hline TACCGATAGTTGCGCTTTTTCA & 22 \\
\hline TTTTCAGGGTGGTTTTTTCTT & 20 \\
\hline AAAGACAAATTAGCAAGTCACCAATGAAACCA & 32 \\
\hline CCAGAATGGAGCCGCCAATCAAGTTTGCC & 29 \\
\hline TTTTTGGATTATTTACAGAA & 20 \\
\hline ATTATAGCGTCGTAATAGTAAAATGTTTTTTT & 31 \\
\hline TAGTCAGAAGCAAAGCGGATTTT & 23 \\
\hline AGGCGAAAATCCTGTTGTCTATCACCCCCGAT & 32 \\
\hline GGGGCGCGCCCAATTCACTAAAGTACGGTGTCACGAGAATAGCTTCAA & 48 \\
\hline GCCGTCACAATATAAAAGAAACCACCAGAAGGAGCGGACTCGTATTACATTTGTCAAATAT & 61 \\
\hline GAAATTGTTATCCGCTCACATTAAATTAATGA & 32 \\
\hline TTTTTCCAAGAACGGGTGCGAACCTTTTT & 29 \\
\hline TGCTCATTCTTATGCGTTAATAAAACGAACTATATTCATTGGCTTTTG & 48 \\
\hline TTTTTAGACTGGCATCAGTTGAGATTTTTTT & 30 \\
\hline AAACGGGGTTTTGCTACATAACGCCAAAAAAGGCT & 35 \\
\hline ACAAAGTATGAGGAAGCTTTGAGGACTAAAGATTTT & 36 \\
\hline TCATCAACAAGGCAAATATGTACCCCGGTTG & 31 \\
\hline
\end{tabular}




\begin{tabular}{|c|c|}
\hline ACAAGAAATAGGAATCCCAATAGCAAGCAAATATAGCAGCATCCTGAA & 48 \\
\hline AGGAGGTGGCGGATAAGTATTAAGAGGCTAAATCCTCTACAGGAG & 45 \\
\hline TTCCGGAATCATAATTTTTT & 20 \\
\hline TTTTGAATGCCAACGGCAGC & 20 \\
\hline TCGAAGATGATGAAACTTTT & 20 \\
\hline TGCCATTCAACAATAGAAAATTCATATGGT & 30 \\
\hline TTTTACTGTAGCCTCAGAACCGCCATTTT & 29 \\
\hline CTGCGCGGCTAACTCACAATTCCACACAACATACGAGTACCGGGGCTCTGTGGGTGTTCAG & 61 \\
\hline AAGCGCATAAATGAAACAGATATAGAAGGCTTAGCAAGCCTTATTACG & 48 \\
\hline CACTCATGAAACCACCTTAAATCAAGATTGAGCGTCTTTTTGTTT & 45 \\
\hline TATTTTTGAGAGATCTGCCATATTTCCTCTACTCAATTGA & 40 \\
\hline GTACTATGGTTGCTTTTTTAAGACACGCAAATT & 32 \\
\hline AATGCAATAGATTAAGGGCTTAGAGCTTATTTTT & 33 \\
\hline GTATAAGCAAATATTTTAGATAAGTAACAACG & 32 \\
\hline AAGGGAACCGGATATTCACTCATCTTTGACCCGTAATGCCATCGGAAC & 48 \\
\hline TGTAGCTCAACATTTACCCTCGAAAGAC & 28 \\
\hline TTTTGTGTAGGTAAAGATTC & 20 \\
\hline TTTTGCCTCAGAGCATAAAGAAAATTAAGCAATAAATTTT & 40 \\
\hline CATAGGTCTGAGAGACAAATCGTCGAATTACC & 32 \\
\hline AGCATGTACGAGAACAATCCGGTATTCTAAGAACGATTTTTCCAGA & 45 \\
\hline GAGAAACATTTAATTTTACAGGTAGAAAG & 29 \\
\hline TTTTAGAGCGGGAGCTAGAT & 20 \\
\hline GTAAGAATAGTTGAAACTTTCGCAAACACCGC & 32 \\
\hline ACCTCGTCATAAACATTTTT & 20 \\
\hline TTCGTAATCATGGTCATCCATCAGTTATAAGT & 32 \\
\hline TTTTGTGTAAAGCCTGGCGG & 20 \\
\hline CCTGCAGCCATAACGGGGTGTCCAGCATCAGC & 32 \\
\hline TTTGCGTATTGGGCGCTTTT & 20 \\
\hline
\end{tabular}




\begin{tabular}{|c|c|}
\hline TTTTCGGGCCGTTTTCACGG & 20 \\
\hline GCCGGGCGCGGTTGCGCCGCTGACCCCTTGTG & 32 \\
\hline TTATACTTAGCACTAAAAAGTTTGTGCCGCCA & 32 \\
\hline GGCACCAAAACCAAAAGTAAGAGCAACACTATAGCAACGTAAATCGCC & 48 \\
\hline CTAGCTGATAAATTAACAGTAGGG & 24 \\
\hline ATACGCAAAGAAAATTATTCATTAAAGGTGAATTTT & 36 \\
\hline AGAGCAAATCCTGTCCAGATACCGACAAAAGGTAATTTT & 39 \\
\hline GCCAGTACGTTATAAGGCGTTAAATAAGAATAAACACAAAT & 41 \\
\hline TTTTTAAACGATGCTGATGG & 20 \\
\hline CCTCAGAGCACAAGAAGAAAAGTAAGCAG & 29 \\
\hline ACAGTTGAGGATCCCCAGATAGAACTGAAAGC & 32 \\
\hline ATAATCAGAAAAGCCCAACATCCACTGTAATA & 32 \\
\hline AAATTATTTGGAAACAGCCATTCGAAAATCGC & 32 \\
\hline ACATTCTGAAGAGTCTCCGCCAGCAGCTCGAA & 32 \\
\hline TTTCATCGAATAATATCCAGCTACAATACTCCAGCAATTTCTTTACAG & 48 \\
\hline TGAATTACCAGTGAATGGAATTACGAGGCATATAGCGAGAGAATCCCC & 48 \\
\hline CGTGCCTGTTCTTCGCATCCAGCGCCGGGTTA & 32 \\
\hline TTAGAGCTATCCTGAGGCTGGTTTCAGGGCGC & 32 \\
\hline TAGTAATAACATCACTTTTT & 20 \\
\hline TAATAAGAAGAGCCACCCTTATTAGCGTT & 29 \\
\hline ATCCAGAACAATATTAGTCCATCAGGAACGGT & 32 \\
\hline TTTTATCGCCATTAAAAATA & 20 \\
\hline TTTTGATTAAGACGCTGAGA & 20 \\
\hline GGAATTAGGTAAATTTTCGGTCATAGCCCCACCGGAACCACCACC & 45 \\
\hline TTTTAGAACGCGAGAAAACTTT & 22 \\
\hline GGGCCTCTTCGCTATTACGTTGTACCTCACCG & 32 \\
\hline TTTTTGCCTGAGTAGAAGAA & 20 \\
\hline GCCAGTGCGATTGACCCACCGCTTCTGGTGCC & 32 \\
\hline
\end{tabular}




\begin{tabular}{|c|c|}
\hline TAAAGTTTAGAACCGCTAATTGTATCGCGGGGTTTAAGTTTGGCCTTG & 48 \\
\hline TTTTATTAAGTTGGGTACGC & 20 \\
\hline ATATATATAAAGCGACGACATCGGCTGTCTTTCCTTATCATTTTTT & 45 \\
\hline CAGATGAATATACAGTTTTT & 20 \\
\hline GACCGTGTGATAAATACAAATTCT & 24 \\
\hline CATAATAATTCGCGTCTTTT & 20 \\
\hline ACAAATTATCATCATATTTT & 20 \\
\hline TCACCGGAAGCATAAATTTT & 20 \\
\hline ATCAAACTTAAATTTCTGGAAGGGCCATATCA & 32 \\
\hline TTCATAGGGTTGAGTGTTTT & 20 \\
\hline GCCCCCTGGTGTATCACCGTACTC & 24 \\
\hline TTCTGAAACATGAAAGTGCCGGCCATTTG & 29 \\
\hline TTTTGGAACCTAAGTCTCTGAATTTTTTTTTTT & 32 \\
\hline TTTTCTTTTTCACAACGGAGATTTGTTTTT & 29 \\
\hline GTTGTACCACCCTCATAAAGGCCGGAGACAG & 31 \\
\hline CCGAGTAAGCCAACAGGGGTACCGCATTGCAA & 32 \\
\hline AGTGTGCTGCAAGGCGTTTT & 20 \\
\hline CCCTGAACAAATAAGAAACGCGAGGCGTT & 29 \\
\hline CATTATACCAGTGATTTGGCATCAGGACGTTGTAACATAAACCAGACG & 48 \\
\hline CAACTAATGCAGACAGAGGGGCAATACTG & 29 \\
\hline CGTTGGTAGTCACGACGCCAGCTGGCGAAAGGGGGATATCGGCCTGCGCATCGGCCAGCTT & 61 \\
\hline AAACGGCGCAAGCTTTGAAGGGCGATCGGTGC & 32 \\
\hline TTTTGTTTCGTCACCAGTACTGTACCGTAAT & 31 \\
\hline CAGTATGTTTATTTTGCGAAGCCCTTTTTTAATTGAGTTCTGAACA & 45 \\
\hline TCAAATCACCATCAATACGCAAGG & 24 \\
\hline CGCTGGCACCACGGGAGACGCAGAAACAGCGG & 32 \\
\hline GAAACAACGCGGTCGCCGCACAGGCGGCCTTTAGTGACTTTCTCCACGTACAGACGCCAGG & 61 \\
\hline TGCTTTCGAGGTGAATCTCCAAAA & 24 \\
\hline
\end{tabular}




\begin{tabular}{|c|c|}
\hline TAGTTGCCAGTTGCGGGAGGTTTTGAAGATCAATAA & 36 \\
\hline AATTACATAGATTTTCAATAACGGATTCGCC & 31 \\
\hline TTTTACCGTTCCAGTAAGCGTCATACATGGCTTCAGTTAAT & 41 \\
\hline TTTTAACAGTACCTTTTACA & 20 \\
\hline CTTTTGCGTTATTTCAATGATATTCAACCGTT & 32 \\
\hline TTTTCATCGGCATATTGACGGCACCACGG & 29 \\
\hline CTCTCACGGAAAAAGAACGGATAAAAACGACG & 32 \\
\hline CGGAATCTCAGGTCTGTTTTAAATATGCATGCGAACGAATCATTG & 45 \\
\hline TGCGGGATAGCAGCGACGAGGCGCAGAGAAACGGCCGCGGTAACGATC & 48 \\
\hline ATTGCGTTTAACAACATTTCAATTACCTGAGCAAAAGGGAGAAACAGGTTTAAGATGATGG & 61 \\
\hline TTCACCAGGTAGCAATGGCCTTGCTGGTAAT & 31 \\
\hline GCCTGTTTGCTTCTGTTACCTTTTTAACGTTAA & 32 \\
\hline CCATTACCAAGGGCGACATCTTTTTCATAGGCAGAAAGAATAGGTTGAG & 48 \\
\hline TCAGCAGCAACCGCAATTTT & 20 \\
\hline CAAATCGTCAGCGTGGTGCCATCCCACGCAA & 31 \\
\hline TTGAGTAAGCCACCCTCAGAACCG & 24 \\
\hline GGAGGGAAGAGCCAGCAATCAGTAGCGACAGACCAGAACCGCCTC & 45 \\
\hline ATAAACAATCCCTTAGTGAATTTATCAAAAT & 31 \\
\hline CACATCCTCAGCGGTGGTATGAGCCGGGTCAC & 32 \\
\hline GCTGCGCAACTGTTGGCAGACCTATTAGAAGG & 32 \\
\hline AAATCAGCTCATTTTTGTGAGCGAATAGGTCA & 32 \\
\hline TTTTAGTAATTCAATCGCAAGACAATTTT & 29 \\
\hline GGAAACCAGGCAAAGCGTACATAAGTGAGTGA & 32 \\
\hline CAAACCCTTTAGTCTTACCAGCAGAAGATAA & 31 \\
\hline CAGTACCATTAGTACCCAGTGCCCGTATAAATTGATGAATTAAAG & 45 \\
\hline AACAGAGGTGAGGCGGCAGACAATTAAAAGGG & 32 \\
\hline TTTTAAGTTACCAGGGTAATTGAGCTTTT & 29 \\
\hline ATTTAGAAGTATTAGATTTT & 20 \\
\hline
\end{tabular}




\begin{tabular}{|c|c|}
\hline CTGATAGCCCTAAAACTTTT & 20 \\
\hline CCGGCAAATCGGCGAAGTGGTGAAGGGATAG & 31 \\
\hline TTTTACCTTGCTGAACCAGG & 20 \\
\hline GGGGTCATTGCAGGCGGGAATTGACTAAAATA & 32 \\
\hline CGCTCACTATCAGACGGTCCGTGAGCCTCCTC & 32 \\
\hline GCAGCAAGCGGTCCACAAGTGTTTTGAGGCCA & 32 \\
\hline CCACCCTCTGTTAGGAAGGATCGTCTTTCCAGCAGACGATTATCAGCT & 48 \\
\hline CTCAAATGTTCAGAAATGGAAGTTTCACGCGCATTACTTCAACTGGCT & 48 \\
\hline ATAACCTTATCAACAAAAATTGTATAACCTCC & 32 \\
\hline ATCGGCCTTAAAGAATAAATCAAAAGAATAGCCCGAGACCAGTGAGGGAGAGGGGTGCCTA & 61 \\
\hline CCAGCTTACGGCTGGAAACGTGCCCGTCTCGT & 32 \\
\hline CTGAGGCCAACGGCTACAGAGGTTTCCATT & 30 \\
\hline AAAACGGTAATCGTTTTTTTT & 20 \\
\hline TGGAGCCGGCCTCCGGGTACATCGACATAAAA & 32 \\
\hline ACGCCAGATGACGGGGCGCCGCTAGCCCCAGC & 32 \\
\hline TTTTACGCATAATGAGAATAGAAAGTTTT & 29 \\
\hline CATGTTTACCAGTCCCTTTT & 20 \\
\hline TTAATTTCATGTTCTATAACTATATGTAAATGCTGATGTCAATAGAATCCTTGACAAAATT & 61 \\
\hline TTTTACTAGAAAAAGCCTGTT & 21 \\
\hline TTAGTTTGCCTGTTTAGGTCATTTTTGCGGATAGGAAGCCGACTATTA & 48 \\
\hline AATAAGTTAGCAAAAACGCAATAATAACGAGAATTAAAAGCCCAA & 45 \\
\hline TTTTTGGCCTTCCTGTATAA & 20 \\
\hline CAGGAAAAACGCTCATACCAGTAAATTTTTTGA & 32 \\
\hline ATATTCACCGCCAGCATTGACAGGCAAAATCA & 32 \\
\hline AACCGTTTCACACGGGAAATACCTACATTTTGACGCTAAACTATCACTTCTTTAACAGGAG & 61 \\
\hline TTTTGCGCTGGCAAGTGTAG & 20 \\
\hline TTTTGGAATTTGTGAGAGAT & 20 \\
\hline
\end{tabular}


Supplementary Table S4. Modified and extended staples from the 5' to the 3' end for the DNAorigami structure used for the tracking experiments (Figure 4).

\begin{tabular}{|c|c|c|}
\hline Sequence $\left(5^{\prime}\right.$ to $\left.3^{\prime}\right)$ & Length [nt] & Function \\
\hline $\begin{array}{l}\text { Biotin-AGAGCCGCAAACAAATGAGACTCCTCAAGA } \\
\text { GATTAGCGGGCAGTAGCA }\end{array}$ & 48 & Biotin for immobilization \\
\hline $\begin{array}{l}\text { Biotin-GAGGGTAGTTGCAGGGTGCTAAACAACTTTCA } \\
\text { CGCCTGGAAAGAG }\end{array}$ & 45 & Biotin for immobilization \\
\hline $\begin{array}{l}\text { Biotin-TACCAGTAACGCTAACAGTTGCTATTTTGCAC } \\
\text { СCСАTCCT }\end{array}$ & 40 & Biotin for immobilization \\
\hline $\begin{array}{l}\text { Biotin-ATAAAAATATCGCGTTCTCCTTTTGATAAGAG } \\
\text { CTATAT }\end{array}$ & 38 & Biotin for immobilization \\
\hline $\begin{array}{l}\text { AGAAACAGCTTTAGAAGGAAGAAAAATCTACGATTTT } \\
\text { AAGCATATAACTTTTAAATGC }\end{array}$ & 58 & Catching site 1 for Cy3B Pointer \\
\hline $\begin{array}{l}\text { GCACCCTCCGTCAGGTACGTTAGTAAATGAATAGTTA } \\
\text { GCGTCAATCATTTTTAAATGC }\end{array}$ & 58 & Catching site 2 for $\mathrm{Cy} 3 \mathrm{~B}$ Pointer \\
\hline $\begin{array}{l}\text { ACGATAAACCTAAAACAAAGAATACACTAAAACATTA } \\
\text { CCCAACAAAGCTTTTTTTTTTCGGGCATTTA-CY3B }\end{array}$ & 67 & Pointer-Cy3B \\
\hline $\begin{array}{l}\text { AGAAACAGCTTTAGAAGGAAGAAAAATCTACGATTTT } \\
\text { AAGCATATAACTTTTAAATGCC }\end{array}$ & 58 & $\begin{array}{l}\text { Catching site } 1 \text { for Atto542 } \\
\text { Pointer }\end{array}$ \\
\hline $\begin{array}{l}\text { GCACCCTCCGTCAGGTACGTTAGTAAATGAATAGTTA } \\
\text { GCGTCAATCATTTTTAAATGCC }\end{array}$ & 58 & $\begin{array}{l}\text { Catching site } 2 \text { for Atto } 542 \\
\text { Pointer }\end{array}$ \\
\hline $\begin{array}{l}\text { ACGATAAACCTAAAACAAAGAATACACTAAAACATTA } \\
\text { CCCAACAAAGCTTTTTTTTTCGGGCATTTA-ATT0542 }\end{array}$ & 67 & Pointer - Atto542 \\
\hline
\end{tabular}




\section{References}

(1) Nickels, P. C.; Wünsch, B.; Holzmeister, P.; Bae, W.; Kneer, L. M.; Grohmann, D.; Tinnefeld, P.; Liedl, T. Molecular Force Spectroscopy with a DNA Origami - Based Nanoscopic Force Clamp. Science 2016, 354 (6310), 305-307.

(2) Vogelsang, J.; Kasper, R.; Steinhauer, C.; Person, B.; Heilemann, M.; Sauer, M.; Tinnefeld, P. A Reducing and Oxidizing System Minimizes Photobleaching and Blinking of Fluorescent Dyes. Angew. Chemie 2008, 47 (29), 5465-5469. https://doi.org/10.1002/anie.200801518.

(3) Balzarotti, F.; Eilers, Y.; Gwosch, K. C.; Gynnå, A. H.; Westphal, V.; Stefani, F. D.; Elf, J.; Hell, S. W. Nanometer Resolution Imaging and Tracking With Minimal Photon Fluxes. Science 2017, 355 (December), 606-612. 